\title{
On the Convergence Rate of the Euler- $\alpha$, an Inviscid Second-Grade Complex Fluid, Model to the Euler Equations
}

\author{
Jasmine S. Linshiz • Edriss S. Titi
}

Received: 9 November 2009 / Accepted: 28 December 2009 / Published online: 14 January 2010

(C) The Author(s) 2010. This article is published with open access at Springerlink.com

\begin{abstract}
We study the convergence rate of the solutions of the incompressible Euler- $\alpha$, an inviscid second-grade complex fluid, equations to the corresponding solutions of the Euler equations, as the regularization parameter $\alpha$ approaches zero. First we show the convergence in $H^{s}, s>n / 2+1$, in the whole space, and that the smooth Euler- $\alpha$ solutions exist at least as long as the corresponding solution of the Euler equations. Next we estimate the convergence rate for two-dimensional vortex patch with smooth boundaries.
\end{abstract}

Keywords Inviscid regularization of Euler equations $\cdot$ Euler- $\alpha \cdot$ Second-grade non-Newtonian fluid · Vortex patch

\section{Introduction}

The equations of motion for a visco-elastic second-grade non-Newtonian complex fluid are given by the system (see, e.g., [28, 29, 74])

$$
\begin{aligned}
& \frac{\partial v}{\partial t}-v \Delta u+(u \cdot \nabla) v+v_{j} \nabla u_{j}+\nabla p=0, \\
& v=\left(1-\alpha^{2} \Delta\right) u, \\
& \nabla \cdot u=0,
\end{aligned}
$$

J.S. Linshiz · E.S. Titi $(\bowtie)$

Department of Computer Science and Applied Mathematics, Weizmann Institute of Science, Rehovot 76100, Israel

e-mail: edriss.titi@weizmann.ac.il

J.S. Linshiz

e-mail: jasmine.tal@weizmann.ac.il

E.S. Titi

Department of Mathematics and Department of Mechanical and Aerospace Engineering, University of California, Irvine, CA 92697-3875, USA

e-mail: etiti@math.uci.edu 


$$
v(x, 0)=v^{i n}(x)
$$

where the fluid velocity field, $v$, and the pressure, $p$, are the unknowns; $v^{i n}$ is the given initial velocity field, $v$ is kinematic viscosity, $\alpha>0$ is a material parameter which represents the elastic response of the fluid, and we use Einstein's summation convention.The inviscid version of this model, i.e., when $v=0$, is mathematically identical to the Euler- $\alpha$ (also known as the Lagrangian-averaged Euler- $\alpha$ ) model, which was independently introduced and derived in the Euler-Poincaré variational framework in [37, 38]. In this variational theory the parameter $\alpha$ is interpreted as a spatial filtering scale of the velocity field $v$.

In $[16-18,30,31]$ the corresponding Navier-Stokes- $\alpha$ (NS- $\alpha$ ) (also known as the viscous Camassa-Holm equations or the Lagrangian-averaged Navier-Stokes- $\alpha$ (LANS- $\alpha$ )) model, which is a regularization of the Navier-Stokes equations (NSE), was obtained by introducing an appropriate $a d$ hoc viscous term into the Euler- $\alpha$ equations, that is, by adding the viscous term $-v \Delta v$, instead of $-v \Delta u$ in (1.1). While the question of global regularity of three-dimensional (3D) viscoelastic model (1.1) is still a challenging open problem, the 3D NS- $\alpha$ model is proven to be globally well posed [31]. The extensive research of the $\alpha$ models (see, e.g., [2, 4, 5, 9, 12-14, 16-18, 20-23, 30-34, 39, 40, 44, 45, 50, 51, 53, 56, $63,67,76])$ stems, on the one hand, from the close agreement of their steady state solutions to averaged empirical data, for a large range of huge Reynolds numbers, for turbulent flows in infinite channels and pipes [16-18]. On the other hand, the $\alpha$-models, for small values of the parameter $\alpha$, can also be viewed as numerical regularizations of the original, Euler or Navier-Stokes, systems. The main practical question arising is that of the applicability of these regularizations to the correct predictions of the underlying flow phenomena. In particular, it becomes important to investigate the problem of convergence of the $\alpha$-models, as the regularization parameter $\alpha$ approaches zero. This problem has been studied in various contexts. In [31] the convergence, as $\alpha \rightarrow 0$, of a subsequence of the weak solutions of the three dimensional (3D) NS- $\alpha$ equations to a Leray-Hopf weak solution of the 3D NSE equations is shown, for the case of periodic boundary conditions. Similar results are also reported in [56] concerning the MHD- $\alpha$ model. In [21, 77] it is shown that the trajectory attractors of the 3D Leray- $\alpha$ and NS- $\alpha$ models converge to the trajectory attractor of weak solutions of the NSE as $\alpha \rightarrow 0$. The authors of [19] obtained a rate of convergence of the solutions of the 3D NS- $\alpha$ equations with periodic boundary to the solutions of the Navier-Stokes equations, as $\alpha \rightarrow 0$, for small initial data in Besov-type function spaces, for which global existence and uniqueness of solutions can be established. Recently, the convergence rates of solutions of various two-dimensional (2D) $\alpha$-regularization models, subject to periodic boundary conditions, toward solutions of the exact Navier-Stokes equations, as $\alpha \rightarrow 0$, have been studied in [14]. For 2D Euler- $\alpha$ regularization the authors of [5] show the convergence of a subsequence of the weak solutions of the Euler- $\alpha$ equations with a distinguished sign vortex sheet initial data (a Radon measure supported on a curve) to a solution of the 2D Euler equations, as $\alpha \rightarrow 0$. We elaborated on this result below. It is worth mentioning that the problem of weak convergence of solutions of the viscous second-grade equations (1.1) with $L^{2}$ initial data to a solution of the Navier-Stokes equations, as $\alpha \rightarrow 0$, is treated in [42, 43]. There is also a result comparing the vorticities of the Euler- $\alpha$ and the NSE equations for $\alpha=\sqrt{v}$ in $\mathbb{R}^{2}[64]$.

In this paper we follow the above mentioned philosophy proposed in [56] and consider the Euler- $\alpha$ model as a numerical inviscid regularization of the Euler equations. We study the convergence rate of the solutions of the 2D and 3D Euler- $\alpha$ equations to the corresponding solutions of the Euler equations for smooth initial data in the whole space, as the regularization parameter $\alpha$ approaches zero. In the $2 \mathrm{D}$ case we also investigate the convergence rate of 
the solutions of the Euler- $\alpha$ to the corresponding solutions of the Euler equations for vortex patch initial data. This program is analogues to the established results concerning the rate of convergence of the NSE to the Euler equations, as the viscosity $v \rightarrow 0$, see [1, 27, 46, 61]. However, in the 2D case there is an advantage of the Euler- $\alpha$ regularization over the NavierStokes equations regularization since the former regularizes the solution by transporting the vorticity with a smooth vector field, and hence it preserves the structure of the vortex patch and vortex sheet while regularizing the motion.

The incompressible Euler equations are given by

$$
\begin{aligned}
& \frac{\partial v}{\partial t}+(v \cdot \nabla) v+\nabla p=0, \\
& \nabla \cdot v=0, \\
& v(x, 0)=v^{i n}(x),
\end{aligned}
$$

where $v$, the fluid velocity field, and $p$, the pressure, are the unknowns, and $v^{\text {in }}$ is the given initial velocity field. For results concerning the Euler equations, see [58, 59], and for recent surveys, see $[6,26]$.

In $\mathbb{R}^{2}$, the vorticity formulation of Euler equations is obtained by taking a curl of (1.2) and is given by

$$
\begin{aligned}
& \frac{\partial q}{\partial t}+(v \cdot \nabla) q=0, \\
& v=K * q, \\
& q(x, 0)=q^{i n}(x),
\end{aligned}
$$

where $K(x)=\frac{1}{2 \pi} \nabla^{\perp} \log |x|, q=\operatorname{curl} v$ is the vorticity, and $q^{i n}$ is the given initial vorticity.

Yudovich [80] obtained the existence and uniqueness of weak solutions of the 2D incompressible Euler equations for initially bounded vorticity (see, also, [3, 49] for an alternative proof, [75] for an improvement with vorticity in a class slightly larger than $L^{\infty}$, and [70] for review of relevant two-dimensional results). In particular, the problem of evolution of vortex patches, where the vorticity is a multiple of the characteristic function of a bounded domain, has a unique global solution, and it was proved in [15] (see also [10]) that $C^{1, \gamma}$, $\gamma>0$, boundaries of the patches remain $C^{1, \gamma}$ for all times. In [27] it was shown that the $L^{2}$ norm of the difference between the solutions of NSE and the corresponding solution of the Euler system for such initial data converges to zero, as the kinematic viscosity $v \rightarrow 0$; even though none of the solutions is in $L^{2}$. The $(v t)^{1 / 2}$ rate of convergence of [27] was improved to $(v t)^{3 / 4}$ in [1], due to the fact that the vorticity of the vortex patch with $C^{1, \gamma}$ boundary is in fact in a Besov space $\dot{B}_{2, \infty}^{1 / 2}$, see also [61] for a simpler proof and an extension to $\mathbb{R}^{3}$.

As we have mentioned above the Euler- $\alpha$ model $[18,25,36-38,60]$ is an inviscid regularization of the Euler equations (1.2), which is given by the system

$$
\begin{aligned}
& \frac{\partial v^{\alpha}}{\partial t}+\left(u^{\alpha} \cdot \nabla\right) v^{\alpha}+v_{j}^{\alpha} \nabla u_{j}^{\alpha}+\nabla p^{\alpha}=0, \\
& v^{\alpha}=\left(1-\alpha^{2} \Delta\right) u^{\alpha}, \\
& \nabla \cdot u^{\alpha}=\nabla \cdot v^{\alpha}=0, \\
& v^{\alpha}(x, 0)=v^{i n, \alpha}(x) .
\end{aligned}
$$


Here $u^{\alpha}$ represents the "filtered" fluid velocity vector, $p^{\alpha}$ is the "filtered" pressure, $\alpha>0$ is a regularization length scale parameter representing the width of the filter. Observe that for $\alpha=0$ one recovers, formally, the Euler equations (1.2). The vorticity of the 2D Euler- $\alpha$ model $q^{\alpha}=\operatorname{curl} v^{\alpha}$ obeys the equations

$$
\begin{aligned}
& \frac{\partial q^{\alpha}}{\partial t}+\left(u^{\alpha} \cdot \nabla\right) q^{\alpha}=0, \\
& u^{\alpha}=K^{\alpha} * q^{\alpha} \\
& q^{\alpha}(x, 0)=q^{i n, \alpha}(x) .
\end{aligned}
$$

The smoothed kernel is $K^{\alpha}=G^{\alpha} * K$, where $G^{\alpha}(x)=\frac{1}{\alpha^{2}} \frac{1}{2 \pi} K_{0}\left(\frac{|x|}{\alpha}\right)$ is the Green function associated with the Helmholtz operator $\left(I-\alpha^{2} \Delta\right)$ (see, e.g., [68]), the function $K_{0}$ is a modified Bessel function of the second kind of order zero (see, e.g., [79]).

The 2D Euler- $\alpha$ equations were studied in [65], where it has been shown that there exists a unique global weak solution to the Euler- $\alpha$ equations with initial vorticity in the space of Radon measures on $\mathbb{R}^{2}$, with a unique Lagrangian flow map describing the evolution of particles. We remark, however, that the question of global existence of weak solutions for the three-dimensional (3D) Euler- $\alpha$ equations is still an open problem. In [45] the global existence of weak solutions is shown for the 3D axisymmetric Euler- $\alpha$ equations without swirl, for initial vorticity being a finite Radon measure with compact support, also, the global existence and uniqueness is established for the compactly supported vorticity in $L^{p}, p>$ $3 / 2$, see also [11].

As in the 2D Euler case, the vortex patch is transported by the flow under evolution of Euler- $\alpha$ equations. The following result is the essence of Theorem A.1 stated below. If the initial vorticity is a multiple of the characteristic function of a simply connected bounded domain $\Omega^{i n}$, with a certain technical condition on the boundary being a simple curve, and the boundary $\partial \Omega^{i n}$ is in either one of the following spaces: Lip, or $C^{1, \beta}, 0 \leq \beta \leq 1$, or $C^{2, \beta}$, $0 \leq \beta<1$, or $C^{n, \beta}, n \geq 3,0<\beta<1$, then the boundary of the vortex patch remains in the corresponding space for all time.

The outline of the paper is as follows. In Sect. 2 we study the convergence rate of the solutions of the Euler- $\alpha$ equations to the solution of the Euler equations for strong solutions that belong to the Sobolev space $H^{m}\left(\mathbb{R}^{n}\right), m>n / 2+1$, for $n=2,3$. We show that interval of existence of Euler- $\alpha$ solutions contains the interval of existence of the corresponding Euler solution, and that the convergence is uniform in time, for time intervals compactly contained in $\left[0, T^{*}\right)$, where $T^{*}$ is the time of existence of the solution of the Euler equations. We also show that in the $H^{m-2}$ norm the solution of the Euler- $\alpha$ equations differs from the solution of the Euler equations by order $\alpha^{2}$. It is worth mentioning that this result corresponds to the $v t$ convergence rate of the solutions of the NSE to the one of the Euler equations, for the inviscid limit of the classical solutions of the NSE equations in the whole space see [24, 46, 47, 61, 62, 69]. The issue of inviscid limit of the NSE in domains with physical boundaries, subject to the no-slip Dirichlet boundary conditions, is a very important open problem, for both theoretical study and applications. The problem emerges first from the boundary layer, which appears because we can not impose a Dirichlet boundary condition for the Euler equation, then the nonlinear advection term of the Navier-Stokes equations may propagate this instability inside the domain. Very few mathematical results are available for this very unstable situation. One of the most striking results in this direction is a theorem of Kato [48], see also [71, 78].

In Sect. 3 we study the convergence for the vortex patch problem. Specifically, we show the convergence, as $\alpha \rightarrow 0$, of the $L^{2}$ norm of the difference between the solutions of the 
Euler- $\alpha$ equations and the solution of the Euler equations for the vortex patch initial data with vorticity being a characteristic function of a simply connected bounded domain with $C^{1, \gamma}, \gamma>0$, boundary, even though neither of the solutions are in $L^{2}$. The convergence rate is of order $\left(\alpha^{2}\right)^{3 / 4}$, which corresponds to the optimal convergence rate of the difference between the solutions of the NSE and the Euler equations which is of order $(v t)^{3 / 4}[1]$.

We remark that, ideally, we would like to compute the rate of convergence in dimensionless units, however due to the absence of typical length scale in $\mathbb{R}^{n}$, the above rates of convergence of the NSE to the Euler equations are given as powers of $(v t)$, which has the units of length square, see, e.g., $[1,27,46,61]$. Similarly, in our case, the rate of convergence involves the parameter $\alpha^{2}$. Observe, that one can artificially cook up a length scale from the initial value, e.g., $\left\|v^{i n}\right\|_{L^{2}}\left\|q^{i n}\right\|_{L^{2}}^{-1}$ in the case of $H^{m}\left(\mathbb{R}^{n}\right), m>n / 2+1$, solutions, or $\left\|q^{i n}\right\|_{L^{1}}^{1 / 2}\left\|q^{i n}\right\|_{L^{\infty}}^{-1 / 2}$ in the $2 \mathrm{D}$ vortex patch case. Contrary to this, if one is interested in bounded domains or domains with periodic boundary conditions, typical length scale will be dictated by the size of the domain, and then the rated of convergence will be expressed as dimensionless quantities.

In the following all the constants $C$ are independent of $\alpha$, and all the $\alpha$ dependencies are spelled explicitly.

\section{Classical Solutions}

In this section we study the convergence rate of the solutions of the Euler- $\alpha$ equations to the solution of the Euler equations for strong solutions that belong to the Sobolev space $H^{m}\left(\mathbb{R}^{n}\right), m>n / 2+1$. For $2 \mathrm{D}$ incompressible flow with initial velocity $v^{\text {in }} \in H^{m}\left(\mathbb{R}^{2}\right)$, $m \geq 3$, the unique solutions of the Euler equations exist globally in time (see, e.g., [58]). Similarly to the Euler case, the Euler- $\alpha$ equations has a unique global solution, since, as in the 2D Euler case, we have an a priori uniform control over the $L^{\infty}$ norm of the vorticity, which implies the global existence, as in the proof of the Beale-Kato-Majda criterion [7]. In $\mathbb{R}^{3}$ only local in time existence of strong solutions of the Euler equations has been shown, see $[46,47,54,55,58]$. The existence and well-posedness of the Euler- $\alpha$ equations for a short time can be easily shown following the classical theory of the Euler equations; see also [41, 57] for an analogue of the Beale-Kato-Majda criterion for the Euler- $\alpha$ model. More precisely, one has the following result:

Proposition 2.1 Let $v^{i n} \in H^{m}\left(\mathbb{R}^{n}\right), m>n / 2+1$. There exists $T^{*}=T^{*}\left(\left\|v^{i n}\right\|_{H^{m}}\right), T^{*} \geq$ $\frac{C}{\left\|v^{i n}\right\|_{H^{m}}}$, such that for any $T<T^{*}$ there exists a unique solution $v \in C\left([0, T] ; H^{m}\left(\mathbb{R}^{n}\right)\right) \bar{\bigcap}$ $A C\left([0, T] ; H^{m-1}\left(\mathbb{R}^{n}\right)\right)$ of the Euler equations (1.2) with initial data $v^{\text {in }}$. In two dimensions the solution exists globally in time. Similar results hold for the Euler- $\alpha$ equations (1.4) with the maximal interval of existence of the three-dimensional Euler- $\alpha$ equations being also dependent on $\alpha$.

In the next theorem we show that the solutions of the Euler- $\alpha$ equations for the $H^{m}$, $m>n / 2+1$, initial data, exist at least as long as the solution of the Euler system exists, and converge, as $\alpha \rightarrow 0$, to the solution of Euler equations. Our proof of this result follows the ideas in [61].

Theorem 2.2 Let $v^{i n}, v^{i n, \alpha} \in H^{m}\left(\mathbb{R}^{n}\right), m>n / 2+1$, and $\left\|v^{i n}-v^{i n, \alpha}\right\|_{H^{m}} \rightarrow 0$, as $\alpha \rightarrow 0$. Let $T^{*}$ be the time of existence of the solution of the Euler system (1.2) $v \in$ 
$C_{l o c}\left(\left[0, T^{*}\right) ; H^{m}\left(\mathbb{R}^{n}\right)\right) \cap A C_{l o c}\left(\left[0, T^{*}\right) ; H^{m-1}\left(\mathbb{R}^{n}\right)\right)$ with initial data $v^{\text {in }}$. Then, for all $0<$ $T<T^{*}$ there exists $0<\bar{\alpha}=\bar{\alpha}\left(v^{i n}, v^{i n, \alpha}, T\right)$ such that for all $\alpha \leq \bar{\alpha}$ there is a unique solution $v^{\alpha} \in C\left([0, T] ; H^{m}\left(\mathbb{R}^{n}\right)\right) \cap A C\left([0, T] ; H^{m-1}\left(\mathbb{R}^{n}\right)\right)$ of the Euler- $\alpha$ equations $(1.4)$ with initial data $v^{\text {in, } \alpha}$. Moreover,

$$
\left\|v^{\alpha}-v\right\|_{L^{\infty}\left([0, T], H^{m}\right)} \rightarrow 0
$$

as $\alpha \rightarrow 0$, and for all $0 \leq t \leq T$

$$
\begin{aligned}
& \left\|\left(v^{\alpha}-v\right)(t)\right\|_{H^{m-2}} \leq\left(\left\|v^{i n}-v^{i n, \alpha}\right\|_{H^{m-2}}+C \alpha^{2} t\right) e^{C t}, \\
& \left\|\left(v^{\alpha}-v\right)(t)\right\|_{H^{m-1}} \leq\left(\left\|v^{i n}-v^{i n, \alpha}\right\|_{H^{m-1}}+C \alpha t\right) e^{C t},
\end{aligned}
$$

where $C=C\left(\left\|v^{i n}\right\|_{H^{m}}, T\right)$ is independent of $\alpha$.

We use the standard $H^{m}\left(\mathbb{R}^{n}\right)$ norm defined by

$$
\|f\|_{H^{m}\left(\mathbb{R}^{n}\right)}^{2}=\int_{\mathbb{R}^{n}}\left(1+|\xi|^{2}\right)^{m}|\hat{f}(\xi)|^{2} d \xi,
$$

where $\hat{f}$ denotes the Fourier transform of $f$.

To prove the theorem we need the following estimates (see, e.g., [46, 59]).

Lemma 2.3 Let $n=2,3$. Let $u, v \in H^{m}\left(\mathbb{R}^{n}\right)$, $\operatorname{div} u=0$, then there exist a constant $C>0$, depending on $m$, such that

$$
\begin{aligned}
& \left|((u \cdot \nabla) v, v)_{H^{m}}\right| \leq C\|\nabla u\|_{H^{m-1}}\|v\|_{H^{m}}^{2} \leq C\|u\|_{H^{m}}\|v\|_{H^{m}}^{2}, \quad m>\frac{n}{2}+1, \\
& \left|((u \cdot \nabla) v, v)_{H^{m}}\right| \leq C\|\nabla u\|_{H^{2}}\|v\|_{H^{m}}^{2} \leq C\|u\|_{H^{3}}\|v\|_{H^{m}}^{2}, \quad m \leq \frac{n}{2}+1 .
\end{aligned}
$$

Let $u \in H^{m}\left(\mathbb{R}^{n}\right), v \in H^{m+1}\left(\mathbb{R}^{n}\right)$, and let $\Psi(u, v)$ be either one of the following bilinear forms: $\Psi(u, v)=u \times(\nabla \times v), \Psi(u, v)=(u \cdot \nabla) v$ or $\Psi(u, v)=v_{j} \nabla u_{j}$, then there exists $a$ constant $C>0$, depending on $m$, such that

$$
\begin{aligned}
\|\Psi(u, v)\|_{H^{m}} & \leq C\|u\|_{H^{m}}\|v\|_{H^{m+1}}, \quad m>\frac{n}{2}, \\
\|\Psi(u, v)\|_{H^{m}} & \leq C\|u\|_{H^{m}}\|v\|_{H^{3}}, \quad m \leq \frac{n}{2},
\end{aligned}
$$

and for $m>\frac{n}{2}+1$

$$
\|\Psi(u, v)\|_{H^{m}} \leq C\left(\|u\|_{H^{m}}\|v\|_{H^{m}}+\|u\|_{L^{\infty}}\|v\|_{H^{m+1}}\right) .
$$

We also use the following lemma

Lemma 2.4 Let $g \in L^{2}\left(\mathbb{R}^{n}\right)$ and $f=\left(1-\alpha^{2} \Delta\right)^{-1} g$. Then

$$
\alpha\left\|(-\Delta)^{1 / 2} f\right\|_{L^{2}} \leq \frac{1}{2}\|g\|_{L^{2}} .
$$

Proof By taking a Fourier transform we have

$$
\hat{f}(\xi)=\frac{\hat{g}(\xi)}{\left(1+\alpha^{2}|\xi|^{2}\right)},
$$


hence

$$
\begin{aligned}
\alpha^{2}\left\|(-\Delta)^{1 / 2} f\right\|_{L^{2}\left(\mathbb{R}^{n}\right)}^{2} & =\int_{\mathbb{R}^{n}}|\hat{g}(\xi)|^{2} \frac{\alpha^{2}|\xi|^{2}}{\left(1+\alpha^{2}|\xi|^{2}\right)^{2}} d \xi \\
& \leq\|g\|_{L^{2}\left(\mathbb{R}^{n}\right)}^{2} \sup _{y \geq 0} \frac{y}{(1+y)^{2}} \leq \frac{1}{4}\|g\|_{L^{2}\left(\mathbb{R}^{n}\right)}^{2} .
\end{aligned}
$$

Next, we prove Theorem 2.2.

Proof First, let us assume that $v, v^{\alpha} \in C\left([0, T] ; H^{m}\left(\mathbb{R}^{n}\right)\right) \cap A C\left([0, T] ; H^{m-1}\left(\mathbb{R}^{n}\right)\right)$ are the solutions of the Euler and the Euler- $\alpha$ systems (1.2) and (1.4) with initial data $v^{i n}$ and $v^{i n, \alpha}$, respectively, on some mutual time interval $[0, T]$, with

$$
\|v\|_{L^{\infty}\left([0, T], H^{m}\right)},\left\|v^{\alpha}\right\|_{L^{\infty}\left([0, T], H^{m}\right)} \leq C\left(T,\left\|v^{i n}\right\|_{H^{m}}\right),
$$

and let us show the convergence rates in $H^{m-2}$ and $H^{m-1}$. In the second part we show that the solutions of the Euler- $\alpha$ equations exist at least up to the time of existence of the solution of Euler equations and satisfy (2.7).

The difference $w^{\alpha}=v-v^{\alpha}$ satisfies the following equation

$$
\begin{aligned}
& \frac{\partial w^{\alpha}}{\partial t}+\left(w^{\alpha} \cdot \nabla\right) v+v_{j} \nabla w_{j}^{\alpha}+\left(u^{\alpha} \cdot \nabla\right) w^{\alpha}+w_{j}^{\alpha} \nabla u_{j}^{\alpha} \\
& \quad-\left(\alpha^{2} \Delta u^{\alpha} \cdot \nabla\right) v-v_{j} \nabla\left(\alpha^{2} \Delta u_{j}^{\alpha}\right)-v_{j} \nabla v_{j}+\nabla\left(p-p^{\alpha}\right)=0, \\
& w^{\alpha}(x, 0)=v^{i n}(x, 0)-v^{i n, \alpha}(x, 0) .
\end{aligned}
$$

Let $k$ be either $m-1$ or $m-2$. Taking the $H^{k}$ inner product of (2.8) with $w^{\alpha}(t)$, we obtain

$$
\frac{1}{2} \frac{d}{d t}\left\|w^{\alpha}\right\|_{H^{k}}^{2} \leq I_{1}+I_{2}+I_{3}+I_{4}
$$

where

$$
\begin{aligned}
& I_{1}=\left|\left(w^{\alpha} \times(\nabla \times v), w^{\alpha}\right)_{H^{k}}\right|, \\
& I_{2}=\left|\left(\left(u^{\alpha} \cdot \nabla\right) w^{\alpha}, w^{\alpha}\right)_{H^{k}}\right|, \\
& I_{3}=\left|\left(w_{j}^{\alpha} \nabla u_{j}^{\alpha}, w^{\alpha}\right)_{H^{k}}\right|, \\
& I_{4}=\alpha^{2}\left|\left(\Delta u^{\alpha} \times(\nabla \times v), w^{\alpha}\right)_{H^{k}}\right|,
\end{aligned}
$$

due to the identity

$$
(b \cdot \nabla) a+a_{j} \nabla b_{j}=-b \times(\nabla \times a)+\nabla(a \cdot b) .
$$

Now, by (2.4)

$$
I_{1} \leq C\|v\|_{H^{m}}\left\|w^{\alpha}\right\|_{H^{k}}^{2},
$$

by (2.3)

$$
I_{2} \leq C\left\|u^{\alpha}\right\|_{H^{m}}\left\|w^{\alpha}\right\|_{H^{k}}^{2} \leq C\left\|v^{\alpha}\right\|_{H^{m}}\left\|w^{\alpha}\right\|_{H^{k}}^{2},
$$


by (2.4)

$$
I_{3} \leq C\left\|u^{\alpha}\right\|_{H^{m}}\left\|w^{\alpha}\right\|_{H^{k}}^{2} \leq C\left\|v^{\alpha}\right\|_{H^{m}}\left\|w^{\alpha}\right\|_{H^{k}}^{2},
$$

and by (2.4)

$$
I_{4} \leq \alpha^{2}\left\|\Delta u^{\alpha}\right\|_{H^{k}}\|v\|_{H^{m}}\left\|w^{\alpha}\right\|_{H^{k}} .
$$

Summing up we get the energy estimate

$$
\frac{1}{2} \frac{d}{d t}\left\|w^{\alpha}\right\|_{H^{k}}^{2} \leq C\left\|w^{\alpha}\right\|_{H^{k}}^{2}\left(\|v\|_{H^{m}}+\left\|v^{\alpha}\right\|_{H^{m}}\right)+\alpha^{2}\left\|\Delta u^{\alpha}\right\|_{H^{k}}\|v\|_{H^{m}}\left\|w^{\alpha}\right\|_{H^{k}} .
$$

Now, for $k=m-2$ we have that

$$
\left\|\Delta u^{\alpha}\right\|_{H^{m-2}} \leq C\left\|u^{\alpha}\right\|_{H^{m}} \leq C\left\|v^{\alpha}\right\|_{H^{m}}
$$

and hence

$$
\frac{1}{2} \frac{d}{d t}\left\|w^{\alpha}\right\|_{H^{m-2}}^{2} \leq C\left\|w^{\alpha}\right\|_{H^{m-2}}^{2}\left(\|v\|_{H^{m}}+\left\|v^{\alpha}\right\|_{H^{m}}\right)+C \alpha^{2}\left\|v^{\alpha}\right\|_{H^{m}}\|v\|_{H^{m}}\left\|w^{\alpha}\right\|_{H^{m-2}},
$$

while for $k=m-1$ we have by (2.6)

$$
\alpha\left\|\Delta u^{\alpha}\right\|_{H^{m-1}} \leq C\left\|(-\Delta)^{1 / 2} v^{\alpha}\right\|_{H^{m-1}} \leq C\left\|v^{\alpha}\right\|_{H^{m}},
$$

and hence

$$
\frac{1}{2} \frac{d}{d t}\left\|w^{\alpha}\right\|_{H^{m-1}}^{2} \leq C\left\|w^{\alpha}\right\|_{H^{m-1}}^{2}\left(\|v\|_{H^{m}}+\left\|v^{\alpha}\right\|_{H^{m}}\right)+C \alpha\left\|v^{\alpha}\right\|_{H^{m}}\|v\|_{H^{m}}\left\|w^{\alpha}\right\|_{H^{m-1}} .
$$

Therefore, by Grönwall lemma and (2.7) we obtain that

$$
\left\|w^{\alpha}(\cdot, t)\right\|_{H^{m-2}} \leq\left(\left\|w^{\alpha}(\cdot, 0)\right\|_{H^{m-2}}+C \alpha^{2} t\right) e^{C t},
$$

and

$$
\left\|w^{\alpha}(\cdot, t)\right\|_{H^{m-1}} \leq\left(\left\|w^{\alpha}(\cdot, 0)\right\|_{H^{m-1}}+C \alpha t\right) e^{C t},
$$

where $C=C\left(\left\|v^{i n}\right\|_{H^{m}}, T\right)$.

Next, we show that the solution of the Euler- $\alpha$ equations (1.4) exists as long as we can solve the Euler system (1.2), and that $v^{\alpha}$ converges to $v$ in $L^{\infty}\left([0, T], H^{m}\right)$, as $\alpha \rightarrow 0$. The proof follows the ideas in [61]. We regularize the initial data by taking $v^{i n, \delta}=\mathcal{F}^{-1}\left(\chi_{|\xi| \leq 1 / \delta}(\xi) \mathcal{F}\left(v^{i n}\right)\right)$ and $v^{i n, \delta, \alpha}=\mathcal{F}^{-1}\left(\chi_{|\xi| \leq 1 / \delta}(\xi) \mathcal{F}\left(v^{i n, \alpha}\right)\right)$, for some $\delta \in\left(0, \delta_{0}\right]$. Let $\alpha^{*}$ be such that $v^{i n, \alpha^{*}} \neq 0$ (if $v^{i n, \alpha}=0$ for all $\alpha$, then the proof is trivial). Since $\| v^{i n, \alpha}-$ $v^{i n} \|_{H^{m}} \rightarrow 0$, as $\alpha \rightarrow 0$, then there exists $\alpha_{0}$ such that $\left\|v^{i n, \alpha}\right\|_{H^{m}} \leq\left\|v^{i n}\right\|_{H^{m}}+\left\|v^{i n, \alpha^{*}}\right\|_{H^{m}}$ for all $\alpha \leq \alpha_{0}$. The regularized initial velocities of the Euler and of the Euler- $\alpha$ equations satisfy, for all $\alpha \leq \alpha_{0}$,

$$
\begin{aligned}
\left\|v^{i n, \alpha, \delta}\right\|_{H^{m}},\left\|v^{i n, \delta}\right\|_{H^{m}} & \leq K, \\
\left\|v^{i n, \alpha, \delta}\right\|_{H^{m+1}},\left\|v^{i n, \delta}\right\|_{H^{m+1}} & \leq \frac{K}{\delta},
\end{aligned}
$$

and for $s \in[0, m]$

$$
\left\|v^{i n, \alpha}-v^{i n, \alpha, \delta}\right\|_{H^{s}},\left\|v^{i n}-v^{i n, \delta}\right\|_{H^{s}} \leq K \delta^{m-s},
$$


where $K=K\left(\left\|v^{i n}\right\|_{H^{m}},\left\|v^{i n, \alpha^{*}}\right\|_{H^{m}}\right)$. In the following we fix $s$ such that $\frac{n}{2}<s<m-1$.

Let $v, v^{\delta}, v^{\alpha}, v^{\alpha, \delta}$ be the corresponding solutions of Euler and Euler- $\alpha$ equations with initial data $v^{i n}$ and $v^{i n, \delta}$, respectively. Notice that, as explained below, the solutions $v, v^{\delta}$, $v^{\alpha}$ and $v^{\alpha, \delta}$ exist on some time interval $\left[0, T_{0}\right], \frac{C}{K} \leq T_{0}<T^{*}$, which is independent of $\alpha$ and $\delta$, and also, for all $t \in\left[0, T_{0}\right]$

$$
\|\varphi(t)\|_{H^{m}},\left\|\varphi^{\delta}(t)\right\|_{H^{m}} \leq C\left(T_{0}, K\right), \quad\left\|\varphi^{\delta}(t)\right\|_{H^{m+1}} \leq \frac{C\left(T_{0}, K\right)}{\delta},
$$

where $\varphi$ denotes $v$ or $v^{\alpha}$, and $\varphi^{\delta}$ denotes either $v^{\delta}$ or $v^{\alpha, \delta}$. Indeed, in $\mathbb{R}^{3}$, writing Euler and Euler- $\alpha$ equations in the vorticity formulation we have that $q=\operatorname{curl} v$ and $q^{\alpha}=\operatorname{curl} v^{\alpha}$ satisfy

$$
\frac{\partial q}{\partial t}+(v \cdot \nabla) q=(q \cdot \nabla) v
$$

and

$$
\frac{\partial q^{\alpha}}{\partial t}+\left(u^{\alpha} \cdot \nabla\right) q^{\alpha}=\left(q^{\alpha} \cdot \nabla\right) u^{\alpha},
$$

respectively. Making the $H^{k}$ estimates (one can use (2.3) and the fact that for $k>3 / 2$, $H^{k}\left(\mathbb{R}^{3}\right)$ is a Banach algebra), due to $m-1>\frac{3}{2}$, we obtain

$$
\begin{aligned}
\frac{d}{d t}\|\psi\|_{H^{m-1}} & \leq C\|\psi\|_{H^{m-1}}^{2}, \\
\frac{d}{d t}\left\|\psi^{\delta}\right\|_{H^{m-1}} & \leq C\left\|\psi^{\delta}\right\|_{H^{m-1}}^{2}, \\
\frac{d}{d t}\left\|\psi^{\delta}\right\|_{H^{m}} & \leq C\left\|\psi^{\delta}\right\|_{H^{m-1}}\left\|\psi^{\delta}\right\|_{H^{m}},
\end{aligned}
$$

where $\psi$ denotes $q$ or $q^{\alpha}$, and $\psi^{\delta}$ denotes $q^{\delta}$ or $q^{\alpha, \delta}$, and hence the solutions exist on a certain interval $\left[0, T_{0}\right]$ depending only on $K$ and $\delta_{0}$, independent of $\alpha$ and $\delta$. Furthermore, we have ${ }^{1}$

$$
\|\psi(t)\|_{H^{m-1}} \leq C\left(T_{0}, K\right), \quad\left\|\psi^{\delta}(t)\right\|_{H^{m}} \leq \frac{C\left(T_{0}, K\right)}{\delta} .
$$

Hence, from the Biot-Savart law, $\varphi=\frac{1}{4 \pi} \int_{\mathbb{R}^{3}} \frac{(x-y)}{|x-y|^{3}} \times \operatorname{curl} \varphi(y) d y$, we obtain (2.10). In $\mathbb{R}^{2}$ the solutions of Euler and Euler- $\alpha$ equations exist for all times, see Proposition 2.1.

${ }^{1}$ It follows that

$$
\|\psi(t)\|_{H^{m-1}} \leq \Psi(t),
$$

where $\Psi$ is the solution of the scalar value initial-value problem $\frac{d}{d t} \Psi(t)=C \Psi^{2}(t)$, where $\Psi(0)$ is the $H^{m-1}$ norm of either one of $q^{i n}, q^{i n, \alpha}, q^{i n, \delta}, q^{i n, \alpha, \delta}, \Psi(0) \leq K$.

Now $\Psi(t)=\frac{\Psi(0)}{1-C t \Psi(0)}$ exists on a certain interval of time $\left[0, T_{0}\right], T_{0}>\frac{1}{C \Psi(0)} \geq \frac{1}{C K}$, obviously independent of $\alpha$ and $\delta$, and we have $\sup _{0 \leq t \leq T_{0}} \Psi(t) \leq \frac{K}{1-C T_{0} K}$. Now, if a solution $\psi(t)$ existing on $\left[0, T^{\alpha, \delta}\right]$, such that $T^{\alpha, \delta}<T_{0}$, then the system can be solved with initial value $\psi^{\delta}\left(T^{\alpha, \delta}\right) \in H^{m-1}$, to continue the solution to $\left[0, T^{\alpha, \delta}+T_{1}^{\alpha, \delta}\right]$ in which (2.11) is true. Iterating this argument we can continue the solution to cover the whole interval $[0, T]$ with the estimate (2.11) throughout. (If the solution cannot be continued at some time $\tilde{T}<T_{0}$, then $\lim \sup _{t \rightarrow \tilde{T}^{-}}\left\|\psi^{\delta}(t)\right\|_{H^{m-1}}=\infty$, a contradiction to (2.11).) 
Now, one can show, following [61], that $v^{\delta}$ is a Cauchy sequence in the Banach space $C\left(\left[0, T_{0}\right], H^{m}\right)$, due to

$$
\begin{aligned}
& \left.\left\|v^{\delta}-v^{\delta^{\prime}}\right\|_{C\left(\left[0, T_{0}\right], H^{m}\right.}\right) \\
& \quad \leq\left(\left\|v^{i n, \delta^{\prime}}-v^{i n, \delta}\right\|_{H^{m}}+\left(\max \left\{\delta, \delta^{\prime}\right\}\right)^{m-s-1} C\left(T_{0},\left\|v^{i n}\right\|_{H^{m}}\right)\right) e^{C\left(T_{0},\left\|v^{i n}\right\|_{H^{m}}\right)} .
\end{aligned}
$$

The limit $v$ of $v^{\delta}$, as $\delta \rightarrow 0$, is the solution of Euler equations, and from (2.12), we have

$$
\left\|v-v^{\delta}\right\|_{L^{\infty}\left(\left[0, T_{0}\right], H^{m}\right)} \leq\left(\left\|v^{i n}-v^{i n, \delta}\right\|_{H^{m}}+C\left(T_{0},\left\|v^{i n}\right\|_{H^{m}}\right) \delta^{m-s-1}\right) C e^{C\left(T_{0},\left\|v^{i n}\right\|_{H^{m}}\right)} .
$$

Next we show that $v^{\alpha, \delta}$ is a Cauchy sequence in $C\left(\left[0, T_{0}\right], H^{m}\right)$, and converges to the solution of Euler- $\alpha$ equations $v^{\alpha}$, as $\delta \rightarrow 0$, and we have

$$
\left\|v^{\alpha}-v^{\alpha, \delta}\right\|_{L^{\infty}\left(\left[0, T_{0}\right], H^{m}\right)} \leq\left(\left\|v^{i n, \alpha}-v^{i n, \alpha, \delta}\right\|_{H^{m}}+C\left(T_{0}, K\right) \delta^{m-s-1}\right) C e^{C\left(T_{0}, K\right)} .
$$

Specifically, in $\mathbb{R}^{3}$ ( $\mathbb{R}^{2}$ case can be done similarly), we first assume that $\delta>\delta^{\prime}$, and denote $\varpi^{\alpha, \delta, \delta^{\prime}}=q^{\alpha, \delta^{\prime}}-q^{\alpha, \delta}, w^{\alpha, \delta, \delta^{\prime}}=v^{\alpha, \delta^{\prime}}-v^{\alpha, \delta}$. We have that

$$
\begin{gathered}
\frac{\partial \varpi^{\alpha, \delta, \delta^{\prime}}}{\partial t}+\left(u^{\alpha, \delta^{\prime}} \cdot \nabla\right) \varpi^{\alpha, \delta, \delta^{\prime}}+\left(\left(\left(1-\alpha^{2} \Delta\right)^{-1} w^{\alpha, \delta, \delta^{\prime}}\right) \cdot \nabla\right) q^{\alpha, \delta} \\
=\left(\varpi^{\alpha, \delta, \delta^{\prime}} \cdot \nabla\right) u^{\alpha, \delta^{\prime}}+\left(q^{\alpha, \delta} \cdot \nabla\right)\left(\left(1-\alpha^{2} \Delta\right)^{-1} w^{\alpha, \delta, \delta^{\prime}}\right) .
\end{gathered}
$$

Using (2.3) and (2.5) we obtain

$$
\begin{aligned}
\frac{d}{d t}\left\|\varpi^{\alpha, \delta, \delta^{\prime}}\right\|_{H^{m-1}}^{2} \leq & C\left(\left\|q^{\alpha, \delta^{\prime}}\right\|_{H^{m-1}}+\left\|q^{\alpha, \delta}\right\|_{H^{m-1}}\right)\left\|\varpi^{\alpha, \delta, \delta^{\prime}}\right\|_{H^{m-1}}^{2} \\
& +C\left\|w^{\alpha, \delta, \delta^{\prime}}\right\|_{L^{\infty}}\left\|q^{\alpha, \delta}\right\|_{H^{m}}\left\|\varpi^{\alpha, \delta, \delta^{\prime}}\right\|_{H^{m-1}} .
\end{aligned}
$$

Now, the difference $w^{\alpha, \delta, \delta^{\prime}}=v^{\alpha, \delta^{\prime}}-v^{\alpha, \delta}$ satisfies the equation (see (2.9))

$$
\frac{\partial w^{\alpha, \delta, \delta^{\prime}}}{\partial t}+\left(\left(1-\alpha^{2} \Delta\right)^{-1} w^{\alpha, \delta}\right) \times\left(\nabla \times v^{\alpha, \delta^{\prime}}\right)+u^{\alpha, \delta} \times\left(\nabla \times w^{\alpha, \delta, \delta^{\prime}}\right)+\nabla\left(\tilde{p}^{\alpha, \delta^{\prime}}-\tilde{p}^{\alpha, \delta}\right)=0,
$$

where $\tilde{p}^{\alpha}, \tilde{p}^{\alpha, \delta}$ are the modified pressure. By (2.3) and (2.4) we obtain that

$$
\frac{1}{2} \frac{d}{d t}\left\|w^{\alpha, \delta, \delta^{\prime}}\right\|_{H^{s}}^{2} \leq C\left(\left\|v^{\alpha, \delta}\right\|_{H^{m}}+\left\|v^{\alpha, \delta^{\prime}}\right\|_{H^{m}}\right)\left\|w^{\alpha, \delta, \delta^{\prime}}\right\|_{H^{s}}^{2} .
$$

Recall, that $\frac{n}{2}<s$, hence, by Sobolev embedding theorem and Grönwall lemma we have

$$
\begin{aligned}
\left\|w^{\alpha, \delta, \delta^{\prime}}\right\|_{L^{\infty}\left(\left[0, T_{0}\right] ; L^{\infty}\right)} & \leq\left\|w^{\alpha, \delta, \delta^{\prime}}\right\|_{L^{\infty}\left([0, T] ; H^{s}\right)} \\
& \leq\left\|w^{\alpha, \delta, \delta^{\prime}}(0)\right\|_{H^{s}} e^{C \int_{0}^{t}\left\|v^{\alpha, \delta}(\tau)\right\|_{H^{m}}+\left\|v^{\alpha, \delta^{\prime}}(\tau)\right\|_{H^{m}} d \tau} \\
& \leq C\left(T_{0}, K\right) \delta^{m-s} e^{C\left(T_{0}, K\right)} .
\end{aligned}
$$


Using also that $\left\|q^{\alpha, \delta}\right\|_{H^{m}} \leq \frac{C\left(T_{0}, K\right)}{\delta}$ we obtain

$$
\begin{aligned}
& \frac{d}{d t}\left\|\varpi^{\alpha, \delta, \delta^{\prime}}\right\|_{H^{m-1}} \\
& \quad \leq C\left(\left\|q^{\alpha, \delta^{\prime}}\right\|_{H^{m-1}}+\left\|q^{\alpha, \delta}\right\|_{H^{m-1}}\right)\left\|\varpi^{\alpha, \delta, \delta^{\prime}}\right\|_{H^{m-1}}+C\left(T_{0}, K\right) \delta^{m-s-1} e^{C\left(T_{0}, K\right)}
\end{aligned}
$$

hence

$$
\left\|\varpi^{\alpha, \delta}\right\|_{L^{\infty}\left(\left[0, T_{0}\right] ; H^{m-1}\right)} \leq\left(\left\|q^{i n, \alpha, \delta^{\prime}}-q^{i n, \alpha, \delta}\right\|_{H^{m-1}}+\delta^{m-s-1} C\left(T_{0}, K\right)\right) e^{C\left(T_{0}, K\right)} .
$$

Making the same estimates for the case $\delta^{\prime} \geq \delta$, we obtain that

$$
\left\|\varpi^{\alpha, \delta}\right\|_{L^{\infty}\left(\left[0, T_{0}\right] ; H^{m-1}\right)} \leq\left(\left\|q^{i n, \alpha, \delta^{\prime}}-q^{i n, \alpha, \delta}\right\|_{H^{m-1}}+\left(\max \left\{\delta, \delta^{\prime}\right\}\right)^{m-s-1} C\left(T_{0}, K\right)\right) e^{C\left(T_{0}, K\right)},
$$

which implies that $\varpi^{\alpha, \delta}$ is a Cauchy sequence in the Banach space $C\left(\left[0, T_{0}\right], H^{m}\right)$, and its limit $v^{\alpha}$, which is the solution of Euler- $\alpha$ equations satisfies (2.14).

It remains to show that $\left\|v^{\delta}(t)-v^{\alpha, \delta}(t)\right\|_{H^{m}}$ converge to zero, as $\alpha$ and $\delta$ converge to zero uniformly in $\left[0, T_{0}\right]$, and then the convergence of $v^{\alpha}$ to $v$ in $L^{\infty}\left(\left[0, T_{0}\right], H^{m}\right)$ follows.

From (2.8) and Lemma 2.3 we have that the difference $w^{\delta}=v^{\delta}-v^{\alpha, \delta}$ satisfy

$$
\begin{aligned}
\frac{d}{d t}\left\|w^{\delta}\right\|_{H^{m}} \leq & C\left(\left\|v^{\delta}\right\|_{H^{m}}+\left\|v^{\alpha, \delta}\right\|_{H^{m}}\right)\left\|w^{\delta}\right\|_{H^{m}}+C\left\|w^{\delta}\right\|_{L^{\infty}}\left(\left\|v^{\delta}\right\|_{H^{m+1}}+\left\|v^{\alpha, \delta}\right\|_{H^{m+1}}\right) \\
& +C \alpha^{2}\left\|\Delta u^{\alpha, \delta}\right\|_{H^{m}}\left\|v^{\delta}\right\|_{H^{m}}+C \alpha^{2}\left\|\Delta u^{\alpha, \delta}\right\|_{L^{\infty}}\left\|v^{\delta}\right\|_{H^{m+1}} .
\end{aligned}
$$

By Sobolev embedding theorem, (2.2) and Grönwall lemma we have that

$$
\begin{aligned}
& \left\|w^{\delta}\right\|_{L^{\infty}\left(\left[0, T_{0}\right], L^{\infty}\left(\mathbb{R}^{n}\right)\right)} \\
& \quad \leq\left\|w^{\delta}\right\|_{L^{\infty}\left(\left[0, T_{0}\right], H^{m-1}\right)} \leq\left(\left\|v^{i n, \delta}-v^{i n, \alpha, \delta}\right\|_{H^{m-1}}+C\left(T_{0}, K\right) \alpha\right) e^{C\left(T_{0}, K\right)} \\
& \quad \leq\left(\left\|v^{i n}-v^{i n, \alpha}\right\|_{H^{m-1}}+C\left(T_{0}, K\right) \alpha\right) e^{C\left(T_{0}, K\right)},
\end{aligned}
$$

by (2.6)

$$
\begin{aligned}
\alpha\left\|\Delta u^{\alpha}\right\|_{H^{m}} & \leq C\left\|v^{\alpha}\right\|_{H^{m+1}}, \\
\alpha\left\|\Delta u^{\alpha, \delta}\right\|_{L^{\infty}} & \leq \alpha\left\|\Delta u^{\alpha, \delta}\right\|_{H^{m-1}} \leq C\left\|v^{\alpha}\right\|_{H^{m}} .
\end{aligned}
$$

Hence

$$
\frac{d}{d t}\left\|w^{\delta}\right\|_{H^{m}} \leq C\left\|w^{\delta}\right\|_{H^{m}}+C\left(\frac{\left\|v^{i n}-v^{i n, \alpha}\right\|_{H^{m-1}}}{\delta}+\frac{\alpha}{\delta}\right) e^{C}+C \frac{\alpha}{\delta},
$$

where $C=C\left(K, T_{0}\right)$. By using Grönwall lemma, first letting $\alpha \rightarrow 0$, and then letting $\delta \rightarrow 0$, while choosing $\delta$ such that $\frac{\left\|v^{i n}-v^{i n, \alpha}\right\|_{H^{m-1}}}{\delta} \rightarrow 0$, we obtain that $\left\|v^{\delta}-v^{\alpha, \delta}\right\|_{L^{\infty}\left(\left[0, T_{0}\right], H^{m}\right)} \rightarrow 0$. Using also (2.13) and (2.14) the convergence of $v^{\alpha}$ to $v$ in $L^{\infty}\left(\left[0, T_{0}\right], H^{m}\right)$ follows.

Now, let the Euler solution exist on $\left[0, T^{*}\right)$, then we can continue the solution of the Euler- $\alpha$ equations up to any $T<T^{*}$ in a finite number of iterations using the above argument. Indeed, we continue the solution from a time interval $\left[0, T_{0}+\ldots+T_{k-1}\right]$ to a time interval $\left[0, T_{0}+\ldots+T_{k}\right]$, by solving the Euler- $\alpha$ equation with the initial data $v^{\alpha}\left(T_{0}+\ldots+T_{k-1}\right), \alpha \leq \min \left\{\alpha_{0}, \ldots, \alpha_{k-1}\right\}$, which converges to $v\left(T_{0}+\ldots+T_{k-1}\right)$ for a time $T_{k} \geq C\left(\left\|v\left(T_{k-1}\right)\right\|_{H^{m}}+\left\|v^{i n, \alpha^{*}}\right\|_{H^{m}}\right)^{-1} \geq C\left(\|v\|_{L^{\infty}\left([0, T], H^{m}\right)}+\left\|v^{i n, \alpha^{*}}\right\|_{H^{m}}\right)^{-1}$. 


\section{The Vortex Patch Case}

In this section we study the convergence, as $\alpha \rightarrow 0$, of the $L^{2}$ norm of the difference between the solutions of the 2D Euler- $\alpha$ equations (1.4) and the solution of the 2D Euler equations (1.2) for the vortex patch initial data with vorticity being a characteristic function of a simply connected bounded domain with $C^{1, \gamma}, \gamma \in(0,1)$, boundary. We show that the convergence rate is of order $\left(\alpha^{2}\right)^{3 / 4}$, which corresponds to the optimal convergence rate of the difference between the solutions of the 2D NSE and the 2D Euler equation which is of order $(v t)^{3 / 4}$.

Yudovich [80] obtained the existence and uniqueness of weak solutions of Euler equations for initial vorticity in $L^{\infty}\left(\mathbb{R}^{2}\right) \cap L^{1}\left(\mathbb{R}^{2}\right)$, in particular, for the problem of evolution of vortex patches, where the initial vorticity $q^{i n}$ is assumed to be proportional to the characteristic function of a bounded domain $\Omega^{i n}, q^{i n}=q_{0} \chi_{\Omega^{i n}}$. Due to the conservation of the vorticity along particle trajectories, the vorticity $q(t)$ remains a characteristic function of an evolving in time domain $\Omega(t)$. For the case where the boundary of the patch $\Omega^{i n}$ belongs to $C^{1, \gamma}, \gamma>0$, it was proved in [15] (see also [10]) that the Euler system has a unique solution $v \in L_{l o c}^{\infty}\left(\mathbb{R}, \operatorname{Lip}\left(\mathbb{R}^{2}\right)\right)$, and $\Omega(t)$ remains a bounded $C^{1, \gamma}$ domain. It was proved in [27] that the $L^{2}$ norm of the difference between the solutions of NSE and the corresponding solution of the Euler system for such initial data converges to zero, as the kinematic viscosity $v \rightarrow 0$ (even though none of the solutions are in $L^{2}$ ). The rate of convergence was improved to $(v t)^{3 / 4}$ in [1], due to the fact that the vorticity of the vortex patch with $C^{1, \gamma}$ boundary is in fact in a Besov space $\dot{B}_{2, \infty}^{1 / 2}$, see also [61] for a simpler proof and an extension to $\mathbb{R}^{3}$.

It has been shown in [65] that there exists a unique global weak solution to the Euler- $\alpha$ equations for initial vorticity in $\mathcal{M}\left(\mathbb{R}^{2}\right)$, the space of finite Radon measures on $\mathbb{R}^{2}$, with a unique Lagrangian flow map describing the evolution of particles. In [5] we show the convergence, as $\alpha \rightarrow 0$, of the weak solutions of Euler- $\alpha$ equations with a distinguished sign initial vorticity in $\mathcal{M}\left(\mathbb{R}^{2}\right) \cap H_{\text {loc }}^{-1}\left(\mathbb{R}^{2}\right)$ (vortex sheet data) to those of the $2 \mathrm{D}$ Euler equations.

Since the solution $q^{\alpha}$ of the Euler- $\alpha$ equation is transported by the smoothed vector field $u^{\alpha}$, then in the Euler- $\alpha$ case the vortex patch is also transported by the flow. The global existence and uniqueness results for the smooth vortex patch evolution under the Euler- $\alpha$ equation can be obtained using arguments similar to those presented in [5] and [58, Chap. 8]. Specifically, if $q^{i n}(x)=q_{0} \chi_{\Omega^{i n}}(x)$ is a multiple of the characteristic function of a simply connected bounded domain $\Omega^{i n}$, and the boundary $\partial \Omega^{i n}$ is in either one of the following spaces: Lip, or $C^{1, \beta}, 0 \leq \beta \leq 1$, or $C^{2, \beta}, 0 \leq \beta<1$, or $C^{n, \beta}, n \geq 3,0<\beta<1$, then the boundary of the vortex patch remains in the same space as $\partial \Omega^{i n}$ for all times. We describe this result in details in Appendix, Sect. A.1.

In studying the convergence rate we use the following results.

Proposition 3.1 Let $q^{i n} \in L^{\infty}\left(\mathbb{R}^{2}\right) \cap L^{1}\left(\mathbb{R}^{2}\right)$. Then there exist unique global solutions $q$ and $q^{\alpha}$ of Euler and Euler- $\alpha$ equations (1.3) and (1.5) respectively. Moreover, the $L^{p}$ norms of $q\left(\right.$ of (1.3)) and $q^{\alpha}\left(\right.$ of (1.5)) are conserved, namely, $\|q(\cdot, t)\|_{L^{p}}=\left\|q^{\alpha}(\cdot, t)\right\|_{L^{p}}=$ $\left\|q^{i n}\right\|_{L^{p}}, 1 \leq p \leq \infty$. In addition, the velocities are bounded uniformly

$$
\|v(\cdot, t)\|_{L^{\infty}},\left\|v^{\alpha}(\cdot, t)\right\|_{L^{\infty}} \leq\left(\left\|q^{i n}\right\|_{L^{1}}\left\|q^{i n}\right\|_{L^{\infty}}\right)^{1 / 2} .
$$

The bounds on the velocities $v$ (of (1.3)) and $v^{\alpha}$ (of (1.5)) are a direct consequence of the Biot-Savart law and the conservation of the $L^{1}$ and $L^{\infty}$ norms of vorticity.

Proposition 3.2 Let $q^{i n}(x)=q_{0} \chi_{\Omega^{i n}}(x)$ be a multiple of the characteristic function of a simply connected bounded domain $\Omega^{\text {in }}$ with $C^{1, \gamma}, \gamma \in(0,1)$, boundary. Then the global 
solutions $v$ and $v^{\alpha}$ of Euler and Euler- $\alpha$ equations (1.3) and (1.5), respectively, are in $L_{\text {loc }}^{\infty}\left(\mathbb{R} ; \operatorname{Lip}\left(\mathbb{R}^{2}\right)\right)$ and for all $\alpha>0, t \in \mathbb{R}$

$$
\begin{aligned}
\|\nabla v(\cdot, t)\|_{L^{\infty}} & \leq\left\|\nabla v^{i n}\right\|_{L^{\infty}} e^{C|t|}, \\
\left\|\nabla v^{\alpha}(\cdot, t)\right\|_{L^{\infty}} & \leq\left\|\nabla v^{i n}\right\|_{L^{\infty}} e^{C|t|},
\end{aligned}
$$

where $C=C\left(q^{\text {in }}\right)$, independent of $\alpha$, and the boundary of the vortex patch remains $C^{1, \gamma}$ for all time.

For the Euler equations this result has been shown in [10]. We adopt their proof to show the uniform, in $\alpha$, bound on $\left\|\nabla v^{\alpha}(\cdot, t)\right\|_{L^{\infty}}$. The vortex-patch problem is reformulated in terms of a scalar function $\varphi^{\alpha}(x, t)$ that defines the patch boundary by $\Omega^{\alpha}(t)=\{\times \in$ $\left.\mathbb{R}^{2} \mid \varphi^{\alpha}(x, t)>0\right\}$ and is convected with the flow by

$$
\begin{aligned}
\frac{\partial \varphi^{\alpha}}{\partial t}+\left(u^{\alpha} \cdot \nabla\right) \varphi^{\alpha} & =0, \\
\varphi^{\alpha}(x, 0) & =\varphi^{i n}(x) .
\end{aligned}
$$

To apply the method of the proof used in [10], the only ingredient we need is to show that $\nabla u^{\alpha}$ is uniformly, in $\alpha$, continuous in the tangential direction of the boundary. First, we recall some properties of the kernel $K^{\alpha}$

$$
K^{\alpha}(x)=\nabla^{\perp} \Psi^{\alpha}(|x|)=\frac{x^{\perp}}{|x|} D \Psi^{\alpha}(|x|),
$$

where

$$
\begin{aligned}
\Psi^{\alpha}(r) & =\frac{1}{2 \pi}\left[K_{0}\left(\frac{r}{\alpha}\right)+\log r\right], \\
D \Psi^{\alpha}(r) & =\frac{d \Psi^{\alpha}}{d r}(r)=\frac{1}{2 \pi}\left[-\frac{1}{\alpha} K_{1}\left(\frac{r}{\alpha}\right)+\frac{1}{r}\right], \\
D^{2} \Psi^{\alpha}(|x|) & =\frac{1}{2 \pi}\left[\frac{1}{\alpha|x|} K_{1}\left(\frac{|x|}{\alpha}\right)+\frac{1}{\alpha^{2}} K_{0}\left(\frac{|x|}{\alpha}\right)-\frac{1}{|x|^{2}}\right], \\
D^{3} \Psi^{\alpha}(r) & =\frac{1}{2 \pi}\left[-\frac{2}{\alpha r^{2}} K_{1}\left(\frac{r}{\alpha}\right)-\frac{1}{\alpha^{2} r} K_{0}\left(\frac{r}{\alpha}\right)-\frac{1}{\alpha^{3}} K_{1}\left(\frac{r}{\alpha}\right)+\frac{2}{r^{3}}\right] .
\end{aligned}
$$

The functions $K_{0}$ and $K_{1}$ denote the modified Bessel functions of the second kind of orders zero and one, respectively. For details on Bessel functions, see, e.g., [79]. Derivatives of $\Psi^{\alpha}$ decay to zero as $\frac{r}{\alpha} \rightarrow \infty$; and as $\frac{r}{\alpha} \rightarrow 0$ satisfy

$$
\begin{gathered}
D \Psi^{\alpha}(r)=-\frac{1}{4 \pi} \frac{r}{\alpha^{2}} \log \frac{r}{\alpha}+O\left(\frac{r}{\alpha^{2}}\right), \\
D^{2} \Psi^{\alpha}(r)=-\frac{1}{4 \pi} \frac{1}{\alpha^{2}} \log \frac{r}{\alpha}+O\left(\frac{1}{\alpha^{2}}\right), \\
D^{3} \Psi^{\alpha}(r)=-\frac{1}{4 \pi} \frac{1}{r \alpha^{2}}+O\left(\frac{r}{\alpha^{4}} \log \frac{r}{\alpha}\right),
\end{gathered}
$$


where the constants in the big $\mathrm{O}$ are independent of $\alpha$. The filtered velocity gradient is given by

$$
\nabla u^{\alpha}(x, t)=\int_{\mathbb{R}^{2}} \nabla K^{\alpha}(x-y) q^{\alpha}(y, t) d y
$$

As in [10], denote by $W$ a divergence free vector field which is tangent to $\partial \Omega, W=\nabla^{\perp} \varphi$. Then

$$
\nabla u^{\alpha}(x) W(x)=\int_{\mathbb{R}^{2}} \nabla K^{\alpha}(x-y) q^{\alpha}(y)[W(x)-W(y)] d y,
$$

and we have the following result corresponding to Corollary 1 and Lemma in the appendix of [10].

Lemma 3.3 For $\gamma \in(0,1)$

$$
\left\|\nabla u^{\alpha} W\right\|_{C^{0, \gamma}} \leq C\left\|\nabla v^{\alpha}\right\|_{L^{\infty}}\|W\|_{C^{0, \gamma}} .
$$

Proof We stress that all the constants $C$ are independent of $\alpha$. We write

$$
\begin{aligned}
& \nabla u^{\alpha}(x) W(x)-\nabla u^{\alpha}(x+h) W(x+h) \\
& =\int_{\mathbb{R}^{2}} \nabla K^{\alpha}(x-y) q^{\alpha}(y)[W(x)-W(y)] d y \\
& \quad-\int_{\mathbb{R}^{2}} \nabla K^{\alpha}(x+h-y) q^{\alpha}(y)[W(x+h)-W(y)] d y \\
& =\int_{|x-y|<2|h|} \nabla K^{\alpha}(x-y) q^{\alpha}(y)[W(x)-W(y)] d y \\
& \quad-\int_{|x-y|<2|h|} \nabla K^{\alpha}(x+h-y) q^{\alpha}(y)[W(x+h)-W(y)] d y \\
& \quad+\int_{|x-y| \geq 2|h|} \nabla K^{\alpha}(x-y) q^{\alpha}(y)[W(x)-W(x+h)] d y \\
& \quad+\int_{|x-y| \geq 2|h|}\left[\nabla K^{\alpha}(x-y)-\nabla K^{\alpha}(x+h-y)\right] q^{\alpha}(y)[W(x+h)-W(y)] d y \\
& =I_{1}+I_{2}+I_{3}+I_{4} .
\end{aligned}
$$

Using the fact that $\left|\nabla K^{\alpha}(x)\right| \leq \frac{C}{|x|^{2}}$ (cf. (3.3), (3.4)), we obtain that $\left|I_{1}\right|,\left|I_{2}\right|$ $\leq C\left\|q^{\alpha}\right\|_{L^{\infty}}\|W\|_{C^{0, \gamma}} h^{\gamma}$, also due to $\left|D^{2} K^{\alpha}(x)\right| \leq \frac{C}{|x|^{3}}$ (cf. (3.3), (3.4)), we have $\left|I_{4}\right| \leq$ $C(\gamma)\left\|q^{\alpha}\right\|_{L^{\infty}}\|W\|_{C^{0, \gamma}} h^{\gamma}$. To bound the term $I_{3}$ we consider two cases $|h| \leq \alpha$ and $|h|>\alpha$ separately. We have

$$
\left|I_{3}\right| \leq\|W\|_{C^{0, \gamma}} h^{\gamma}|J|,
$$

where $J=\int_{|x-y| \geq 2|h|} \nabla K^{\alpha}(x-y) q^{\alpha}(y) d y$. First, let $|h| \leq \alpha$, write $J$ as

$$
\begin{aligned}
J & =\int_{\mathbb{R}^{2}} \nabla K^{\alpha}(x-y) q^{\alpha}(y) d y-\int_{|x-y|<2|h|} \nabla K^{\alpha}(x-y) q^{\alpha}(y) d y \\
& =\nabla u^{\alpha}(x)-J_{1} .
\end{aligned}
$$


By (3.3)-(3.5), we obtain

$$
\begin{aligned}
\left|J_{1}\right| & \leq\left\|q^{\alpha}\right\|_{L^{\infty}} \int_{|x-y|<2|h|}\left|\nabla K^{\alpha}(x-y)\right| d y \\
& \leq\left\|q^{\alpha}\right\|_{L^{\infty}} \int_{\frac{|x-y|}{\alpha}<2}\left(\frac{1}{4 \pi} \frac{1}{\alpha^{2}}\left|\log \frac{|x-y|}{\alpha}\right|+\frac{C}{\alpha^{2}}\right) d y \\
& \leq C\left\|q^{\alpha}\right\|_{L^{\infty}} .
\end{aligned}
$$

Now, let $|h|>\alpha$, then we write

$$
\begin{aligned}
J & =\int_{|x-y| \geq 2|h|}\left(\nabla K^{\alpha}(x-y)-\nabla K(x-y)\right) q^{\alpha}(y) d y+\int_{|x-y| \geq 2|h|} \nabla K(x-y) q^{\alpha}(y) d y \\
& =J_{2}+J_{3} .
\end{aligned}
$$

A bound for $\left|J_{3}\right|$ is obtained by using a lemma due to Cotlar (see [72, p. 291])

$$
\begin{aligned}
\left|J_{3}\right| & \leq C\left(\| \text { p.v. } \int_{\mathbb{R}^{2}} \nabla K(x-y) q^{\alpha}(y) d y\left\|_{L^{\infty}}+\right\| q^{\alpha} \|_{L^{\infty}}\right) \\
& =C\left(\left\|\nabla v^{\alpha}\right\|_{L^{\infty}}+\left\|q^{\alpha}\right\|_{L^{\infty}}\right) .
\end{aligned}
$$

We bound $\left|J_{2}\right|$ using the facts that $K_{0}, K_{1} \geq 0$ satisfy $\int_{\mathbb{R}^{2}} \frac{1}{\alpha^{2}} K_{0}\left(\frac{|x|}{\alpha}\right) d x=2 \pi$, $\int_{|x|>2 \alpha} \frac{1}{\alpha|x|} K_{1}\left(\frac{|x|}{\alpha}\right) d x=\pi K_{0}(2)$, and we obtain

$$
\begin{aligned}
\left|J_{2}\right| & \leq C\left\|q^{\alpha}\right\|_{L^{\infty}} \int_{|x-y|>2 \alpha}\left[\frac{1}{\alpha|x-y|} K_{1}\left(\frac{|x-y|}{\alpha}\right)+\frac{1}{\alpha^{2}} K_{0}\left(\frac{|x-y|}{\alpha}\right)\right] d y \\
& \leq C\left\|q^{\alpha}\right\|_{L^{\infty}} .
\end{aligned}
$$

To conclude, in both cases we have

$$
\left|I_{3}\right| \leq C\|W\|_{C^{0, \gamma}} h^{\gamma}\left(\left\|\nabla v^{\alpha}\right\|_{L^{\infty}}+\left\|q^{\alpha}\right\|_{L^{\infty}}\right)
$$

Next we briefly recall the definition of homogeneous Besov spaces. Let $\mathcal{S}\left(\mathbb{R}^{n}\right)$ be the Schwartz space and denote by $\mathcal{Z}^{\prime}\left(\mathbb{R}^{n}\right)$ the dual space of $\mathcal{Z}\left(\mathbb{R}^{n}\right)=\left\{f \in S\left(\mathbb{R}^{n}\right): D^{\beta} \hat{f}(0)=\right.$ 0 for every multi-index $\beta \in \mathbb{N}^{n}$, it can also be identified as the quotient space of $\mathcal{S}^{\prime} / \mathcal{P}$, where $\mathcal{P}$ is the collection of all polynomials. Here $\hat{\varphi}$ and $\mathcal{F}(\varphi)$ denote the Fourier transform of $\varphi$ in $\mathbb{R}^{n}$.

We recall the Littlewood-Paley decomposition. Choose a radial function $\varphi \in \mathcal{S}$ such that $\hat{\varphi} \in C_{0}^{\infty}\left(\mathbb{R}^{n} \backslash\{0\}\right)$ satisfies supp $\hat{\varphi} \subset\left\{\frac{3}{4} \leq|\xi| \leq \frac{8}{3}\right\}$ and $\sum_{q=-\infty}^{\infty} \hat{\varphi}_{q}(\xi)=1$ for $\xi \neq 0$, where $\hat{\varphi}_{q}(\xi)=\hat{\varphi}\left(2^{-q} \xi\right)$, that is, $\varphi_{q}(x)=2^{q n} \varphi\left(2^{q} x\right)$. For $q \in \mathbb{Z}$ one defines the dyadic blocks by $\Delta_{q} f=\mathcal{F}^{-1}\left(\mathcal{F}\left(\varphi_{q}\right) \mathcal{F}(f)\right)=\varphi_{q} * f$. The formal decomposition $f=\sum_{q=-\infty}^{\infty} \Delta_{q} f$ holds true modulo polynomials and is called the homogeneous Littlewood-Paley decomposition. For $s \in \mathbb{R}, 1 \leq r \leq \infty$, the homogeneous Besov space is defined as

$$
\dot{B}_{2, r}^{s}=\left\{f \in \mathcal{Z}^{\prime}\left(\mathbb{R}^{n}\right):\|f\|_{\dot{B}_{2, r}^{s}}<\infty\right\},
$$


where $\|f\|_{\dot{B}_{2, r}^{s}}=\left(\sum_{q=-\infty}^{\infty} 2^{r s q}\left\|\Delta_{q} f\right\|_{L^{2}}^{r}\right)^{1 / r}$ for $r \in[1, \infty)$ and $\|f\|_{\dot{B}_{2, \infty}^{s}}=\sup _{q \in \mathbb{Z}}\left(2^{s q} \times\right.$ $\left\|\Delta_{q} f\right\|_{L^{2}}$ ). For the detailed description of Besov space see, e.g., [8, 52, 66, 73].

We use the following results:

Lemma 3.4 [61] For $0<\beta<1$

$$
\|f\|_{\dot{B}_{2,1}^{1-\beta}} \leq C\|f\|_{L^{2}}^{\beta}\|\nabla f\|_{L^{2}}^{1-\beta} .
$$

Lemma 3.5 Let $s \in \mathbb{R}, \beta \geq 0$. There exist constants $c$ and $C$ such that

$$
c^{-\beta}\|f\|_{B_{2, \infty}^{s+\beta}} \leq\left\|(-\Delta)^{\frac{\beta}{2}} f\right\|_{B_{2, \infty}^{s}} \leq C^{\beta}\|f\|_{B_{2, \infty}^{s+\beta}}
$$

Proof This lemma follows directly from definition of the Besov spaces since

$$
\left\|\Delta_{q}\left((-\Delta)^{\frac{\beta}{2}} f\right)\right\|_{L^{2}}=\left\|\mathcal{F}\left(\varphi_{q}\right) \mathcal{F}\left((-\Delta)^{\frac{\beta}{2}} f\right)\right\|_{L^{2}}=\left\|\hat{\varphi}\left(2^{-q} \xi\right)|\xi|^{\beta} \hat{f}(\xi)\right\|_{L^{2}}
$$

and because $\hat{\varphi}\left(2^{-q} \xi\right)$ is supported in $\left\{\xi \in \mathbb{R}^{n}: \frac{3}{4} 2^{q} \leq|\xi| \leq \frac{8}{3} 2^{q}\right\}$.

Lemma 3.6 Let $0 \leq \beta \leq 2, g \in \dot{B}_{2, r}^{\beta}$ and $f=\left(1-\alpha^{2} \Delta\right)^{-1} g$. Then

$$
\alpha^{\beta}\left\|(-\Delta)^{\beta / 2} f\right\|_{\dot{B}_{2, r}^{\beta}} \leq\|g\|_{\dot{B}_{2, r}^{\beta}}
$$

Proof We have

$$
\begin{aligned}
\alpha^{\beta}\left\|\Delta_{q}\left((-\Delta)^{\beta / 2} f\right)\right\|_{L^{2}} & =\alpha^{\beta}\left\|\mathcal{F}\left(\varphi_{q}\right) \mathcal{F}\left((-\Delta)^{\frac{\beta}{2}} f\right)\right\|_{L^{2}} \\
& =\alpha^{\beta}\left\|\mathcal{F}\left(\varphi_{q}\right) \frac{|\xi|^{\beta} \hat{g}(\xi)}{1+\alpha^{2}|\xi|^{2}}\right\|_{L^{2}} \\
& =\left(\int_{\mathbb{R}^{n}} \frac{\left(\alpha^{2}|\xi|^{2}\right)^{\beta}}{\left(1+\alpha^{2}|\xi|^{2}\right)^{2}}\left(\hat{\varphi}\left(2^{-q} \xi\right) \hat{g}(\xi)\right)^{2} d \xi\right)^{1 / 2} \\
& \leq\left(\left(\sup _{y \geq 0} \frac{y^{\beta}}{(1+y)^{2}}\right) \int_{\mathbb{R}^{n}}\left(\hat{\varphi}\left(2^{-q} \xi\right) \hat{g}(\xi)\right)^{2} d \xi\right)^{1 / 2} \\
& \leq\left\|\Delta_{q} g\right\|_{L^{2}} .
\end{aligned}
$$

From which (3.8) follows.

The two following propositions show that the vortex patch vorticity, with $C^{1, \gamma}$ boundary, evolving under Euler- $\alpha$ equations is in a homogeneous Besov space $\dot{B}_{2, \infty}^{1 / 2}$.

Proposition 3.7 [61] If $\Omega$ is a bounded domain with $C^{1, \gamma}, \gamma>0$ boundary, then $\chi_{\Omega} \in \dot{B}_{2, \infty}^{1 / 2}$.

Proposition 3.8 Let $v \in L^{\infty}\left([0, T] ; \operatorname{Lip}\left(\mathbb{R}^{2}\right)\right)$ with $\operatorname{div} v=0$. Let $\varphi$ solve

$$
\frac{\partial \varphi}{\partial t}+(v \cdot \nabla) \varphi=0
$$




$$
\varphi(x, 0)=\varphi^{i n}(x)
$$

with $\varphi^{i n} \in \dot{B}_{2, \infty}^{1 / 2}$. Then

$$
\|\varphi(\cdot, t)\|_{\dot{B}_{2, \infty}^{1 / 2}} \leq\left\|\varphi^{i n}\right\|_{\dot{B}_{2, \infty}^{1 / 2}} e^{C \int_{0}^{t}\|\nabla v(\cdot, \tau)\|_{L^{\infty} d \tau}}
$$

This result is a straightforward adaptation of the Proposition 3.1 of [1] to the homogeneous Besov spaces.

Propositions 3.2, 3.7 and 3.8 imply the following result:

Corollary 3.9 $\operatorname{Let}^{i n}(x)=q_{0} \chi_{\Omega^{i n}}(x)$ be a multiple of the characteristic function of a simply connected bounded domain $\Omega^{\text {in }}$ with $C^{1, \gamma}, \gamma>0$, boundary. Then the global solutions $q$ and $q^{\alpha}$ of Euler and Euler- $\alpha$ equations (1.3) and (1.5), respectively, satisfy for all $\alpha>0$, $t \in \mathbb{R}^{+}$

$$
\begin{gathered}
\|q(\cdot, t)\|_{\dot{B}_{2, \infty}^{1 / 2}} \leq\left\|q^{i n}\right\|_{\dot{B}_{2, \infty}^{1 / 2}} e^{C \int_{0}^{t}\|\nabla v(\cdot, \tau)\|_{L^{\infty}} d \tau}, \\
\left\|q^{\alpha}(\cdot, t)\right\|_{\dot{B}_{2, \infty}^{1 / 2}} \leq\left\|q^{i n}\right\|_{\dot{B}_{2, \infty}^{1 / 2}} e^{C \int_{0}^{t}\left\|\nabla u^{\alpha}(\cdot, \tau)\right\|_{L^{\infty}} d \tau} .
\end{gathered}
$$

Next we state a lemma that play an important role in estimating the convergence rate.

Lemma 3.10 Let $q^{i n}=q_{0} \chi_{\Omega^{i n}}$, with $\Omega^{\text {in }}$ being a simply connected bounded domain $\Omega^{i n}$ with $C^{1, \gamma}$ boundary, $\gamma \in(0,1)$. Then for all $T \in[0, \infty)$ the solution $u^{\alpha}$ of the Euler- $\alpha$ equations (1.4) satisfies

$$
\alpha^{1 / 2}\left\|\Delta u^{\alpha}(\cdot, t)\right\|_{L^{2}} \leq C\left\|q^{\alpha}\right\|_{L^{\infty}\left([0, T], \dot{B}_{2, \infty}^{1 / 2}\right)}
$$

for all $t \in[0, T]$.

Proof Let $0<\beta<\frac{1}{2}$. Since $\left(\dot{B}_{2,1}^{-\beta}\right)^{\prime}=\dot{B}_{2, \infty}^{\beta}$, for $\beta \in \mathbb{R}$ (see, e.g., [73]), we have

$$
\alpha^{\beta+1 / 2}\left\|\Delta u^{\alpha}\right\|_{L^{2}}^{2}=\alpha^{\beta+1 / 2} \int_{\mathbb{R}^{n}} \Delta u^{\alpha}(x) \cdot \Delta u^{\alpha}(x) d x \leq C \alpha^{\beta+1 / 2}\left\|\Delta u^{\alpha}\right\|_{\dot{B}_{2, \infty}^{\beta}}\left\|\Delta u^{\alpha}\right\|_{\dot{B}_{2,1}^{-\beta}} .
$$

We stress that all the constants $C$ are independent of $\alpha$ and $\beta$. Now, by (3.8) and (3.7)

$$
\alpha^{\beta+1 / 2}\left\|\Delta u^{\alpha}\right\|_{\dot{B}_{2, \infty}^{\beta}} \leq\left\|(-\Delta)^{3 / 4-\beta / 2} v^{\alpha}\right\|_{\dot{B}_{2, \infty}^{\beta}} \leq C\left\|(-\Delta)^{1 / 2} v^{\alpha}\right\|_{\dot{B}_{2, \infty}^{1 / 2}} \leq C\left\|q^{\alpha}\right\|_{\dot{B}_{2, \infty}^{1 / 2}},
$$

and by (3.6)

$$
\begin{aligned}
\left\|\Delta u^{\alpha}\right\|_{\dot{B}_{2,1}^{-\beta}} & \leq C\left\|\nabla u^{\alpha}\right\|_{L^{2}}^{\beta}\left\|\Delta u^{\alpha}\right\|_{L^{2}}^{1-\beta} \\
& \leq C\left\|q^{i n}\right\|_{L^{2}}^{\beta}\left\|\Delta u^{\alpha}\right\|_{L^{2}}^{1-\beta} .
\end{aligned}
$$

Summing up we have

$$
\alpha^{\beta+1 / 2}\left\|\Delta u^{\alpha}\right\|_{L^{2}}^{2} \leq C\left\|q^{\alpha}\right\|_{\dot{B}_{2, \infty}^{1 / 2}}\left\|q^{i n}\right\|_{L^{2}}^{\beta}\left\|\Delta u^{\alpha}\right\|_{L^{2}}^{1-\beta},
$$


hence

$$
\alpha^{\frac{\beta+1 / 2}{1+\beta}}\left\|\Delta u^{\alpha}\right\|_{L^{2}} \leq C\left\|q^{\alpha}\right\|_{\dot{B}_{2, \infty}^{1 / 2}}^{1 /(1+\beta)}\left\|q^{i n}\right\|_{L^{2}}^{\beta /(1+\beta)},
$$

and taking the limit as $\beta \rightarrow 0$ we obtain

$$
\alpha^{\frac{1}{2}}\left\|\Delta u^{\alpha}\right\|_{L^{2}} \leq C\left\|q^{\alpha}\right\|_{\dot{B}_{2, \infty}^{1 / 2}} .
$$

In the next theorem we show that the solution of the Euler- $\alpha$ equations differs from the solution of the Euler equations by order $\left(\alpha^{2}\right)^{3 / 4}$, both having the same vortex patch as initial data.

Theorem 3.11 Let $q^{i n}=q_{0} \chi_{\Omega^{i n}}$, where $\chi_{\Omega^{i n}}$ is a characteristic function of a simply connected bounded domain $\Omega^{\text {in }}$ with $C^{1, \gamma}, \gamma \in(0,1)$, boundary, and let $v, v^{\alpha}$ be the solutions of the Euler and the Euler- $\alpha$ equations (1.2) and (1.4), respectively, with initial data $q^{i n}$. Then the difference $w^{\alpha}=v-v^{\alpha}$ is square-integrable and obeys the estimate

$$
\left\|w^{\alpha}(\cdot, t)\right\|_{L^{2}} \leq \alpha^{3 / 2} C V(t)\left\|q^{i n}\right\|_{\dot{B}_{2, \infty}^{1 / 2}} e^{C V(t)},
$$

where

$$
V(t)=\int_{0}^{t}\left(\|\nabla v(\cdot, \tau)\|_{L^{\infty}}+\left\|\nabla u^{\alpha}(\cdot, \tau)\right\|_{L^{\infty}}\right) d \tau .
$$

In particular, there exists a constant $C=C\left(q^{\text {in }}\right)$ such that

$$
\left\|w^{\alpha}(\cdot, t)\right\|_{L^{2}} \leq \alpha^{3 / 2} C e^{C e^{C t}} .
$$

Proof The difference $w^{\alpha}=v-v^{\alpha}$ satisfies the following equation

$$
\begin{aligned}
& \frac{\partial w^{\alpha}}{\partial t}+\left(w^{\alpha} \cdot \nabla\right) v+v_{j} \nabla w_{j}^{\alpha}+\left(u^{\alpha} \cdot \nabla\right) w^{\alpha}+w_{j}^{\alpha} \nabla u_{j}^{\alpha} \\
& \quad-\left(\alpha^{2} \Delta u^{\alpha} \cdot \nabla\right) v-v_{j} \nabla\left(\alpha^{2} \Delta u_{j}^{\alpha}\right)-v_{j} \nabla v_{j}+\nabla\left(p-p^{\alpha}\right)=0 .
\end{aligned}
$$

We remark that $v$ and $v^{\alpha}$, obtained from the vortex patch vorticity by convolution with the Biot-Savart kernel are not in $L^{2}$, however, the difference $w^{\alpha} \in L^{\infty}\left([0, T], L^{2}\left(\mathbb{R}^{2}\right)\right)$, since curl $w^{\alpha}$ is compactly supported and $\int_{\mathbb{R}^{2}} \operatorname{curl} w^{\alpha}(x, t) d x=\int_{\mathbb{R}^{2}}\left(q^{i n}-q^{i n, \alpha}\right)(x) d x=$ 0 (this could be seen by using an asymptotic expansion of the kernel, see, e.g., [58], p. 321). Also, all the terms in (3.10) are in $L^{\infty}\left([0, T], L^{2}\left(\mathbb{R}^{2}\right)\right)$, since the velocities are in $L^{\infty}\left([0, T], L^{\infty}\left(\mathbb{R}^{2}\right)\right)$, and their gradients, as well as gradients of the pressures, are in $L^{\infty}\left([0, T], L^{2}\left(\mathbb{R}^{2}\right)\right)$.

We take an $L^{2}$-inner product of (3.10) with $w^{\alpha}(x, t)$ and obtain that

$$
\frac{1}{2} \frac{d}{d t}\left\|w^{\alpha}(\cdot, t)\right\|_{L^{2}}^{2} \leq I_{1}+I_{2}+I_{3},
$$

where

$$
\begin{aligned}
& I_{1}=\left|\left(w_{j}^{\alpha} \nabla u_{j}^{\alpha}, w^{\alpha}\right)\right|, \\
& I_{2}=\alpha^{2}\left|\left(\left(\Delta u^{\alpha} \cdot \nabla\right) v, w^{\alpha}\right)\right|+\alpha^{2}\left|\left(\left(w^{\alpha} \cdot \nabla\right) v, \Delta u^{\alpha}\right)\right|,
\end{aligned}
$$




$$
I_{3}=\left|\left(\nabla\left(p-p^{\alpha}\right), w^{\alpha}\right)\right|,
$$

here we used the identity, for $h$ divergence free,

$$
\left((f \cdot \nabla) g+g_{j} \nabla f_{j}, h\right)=((f \cdot \nabla) g, h)-((h \cdot \nabla) g, f) .
$$

The first term is estimated by

$$
I_{1} \leq\left\|w^{\alpha}\right\|_{L^{2}}^{2}\left\|\nabla u^{\alpha}\right\|_{L^{\infty}}
$$

For the second term by (3.9) we obtain

$$
\begin{aligned}
I_{2} & \leq \alpha^{2}\left\|\Delta u^{\alpha}\right\|_{L^{2}}\|\nabla v\|_{L^{\infty}}\left\|w^{\alpha}\right\|_{L^{2}} \\
& \leq C \alpha^{3 / 2}\left\|q^{\alpha}\right\|_{L^{\infty}\left([0, T], \dot{B}_{2, \infty}^{1 / 2}\right)}\|\nabla v\|_{L^{\infty}}\left\|w^{\alpha}\right\|_{L^{2}} .
\end{aligned}
$$

It remains to estimate the third term. We remark that the pressure is determined uniquely up to a constant, and in order to ensure that $p \in L^{2}$, we require a side condition $\int_{\mathbb{R}^{2}} p(x) d x=0$. Notice that the mean free pressure of Euler equations satisfy

$$
p=R_{i} R_{j}\left(v_{i} v_{j}\right)
$$

where $R_{i}=(-\Delta)^{-1 / 2} \frac{\partial}{\partial x_{i}}$ is the Riesz transform (see, e.g., [35]), hence by the properties of the Riesz transform and the Sobolev embedding theorem we have

$$
\|p\|_{L^{2}}=\left\|v_{i} v_{j}\right\|_{L^{2}} \leq\|v\|_{L^{4}}^{2} \leq C\|v\|_{W^{1,4 / 3}}^{2} \leq C\left\|q^{i n}\right\|_{L^{4 / 3}}^{2} .
$$

For the Euler- $\alpha$ equation we have

$$
-\Delta p^{\alpha}=\frac{\partial}{\partial x_{i}} \frac{\partial}{\partial x_{j}}\left(u_{i}^{\alpha} v_{j}^{\alpha}\right)+\frac{1}{2} \frac{\partial^{2}}{\partial x_{i}^{2}}\left(u_{j}^{\alpha}\right)^{2}-\frac{\partial}{\partial x_{i}}\left(\alpha^{2} \Delta u_{j}^{\alpha} \frac{\partial}{\partial x_{i}} u_{j}^{\alpha}\right),
$$

we write

$$
p^{\alpha}=p_{1}^{\alpha}+p_{2}^{\alpha},
$$

where the mean free pressure $p_{1}^{\alpha}, \int_{\mathbb{R}^{2}} p_{1}^{\alpha}(x) d x=0$, satisfies

$$
-\Delta p_{1}^{\alpha}=\frac{\partial}{\partial x_{i}} \frac{\partial}{\partial x_{j}}\left(u_{i}^{\alpha} v_{j}^{\alpha}\right)+\frac{1}{2} \frac{\partial^{2}}{\partial x_{i}^{2}}\left(u_{j}^{\alpha}\right)^{2},
$$

and $p_{2}^{\alpha}$ satisfies

$$
-\Delta p_{2}^{\alpha}=-\alpha^{2} \frac{\partial}{\partial x_{i}}\left(\Delta u_{j}^{\alpha} \frac{\partial}{\partial x_{i}} u_{j}^{\alpha}\right) .
$$

As described above for the pressure of the Euler equations, we have

$$
\left\|p_{1}^{\alpha}\right\|_{L^{2}} \leq C\left\|q^{i n}\right\|_{L^{4 / 3}}^{2} .
$$

For the $p_{2}^{\alpha}$ we have

$$
\left\|\nabla p_{2}^{\alpha}\right\|_{L^{2}}=\alpha^{2}\left\|\Delta u_{j}^{\alpha} \frac{\partial}{\partial x_{i}} u_{j}^{\alpha}\right\|_{L^{2}} \leq \alpha^{2}\left\|\Delta u^{\alpha}\right\|_{L^{2}}\left\|\nabla u^{\alpha}\right\|_{L^{\infty}} .
$$


Hence, using also (3.9), we obtain

$$
I_{3} \leq\left\|\nabla p_{2}^{\alpha}\right\|_{L^{2}}\left\|w^{\alpha}\right\|_{L^{2}} \leq C \alpha^{3 / 2}\left\|q^{\alpha}\right\|_{L^{\infty}\left([0, T], \dot{B}_{2, \infty}^{1 / 2}\right)}\left\|\nabla u^{\alpha}\right\|_{L^{\infty}}\left\|w^{\alpha}\right\|_{L^{2}} .
$$

From the above and (3.11), and by using Grönwall lemma, we obtain

$$
\left\|w^{\alpha}(\cdot, t)\right\|_{L^{2}} \leq C \alpha^{3 / 2}\left\|q^{i n}\right\|_{\dot{B}_{2, \infty}^{1 / 2}} V(t) e^{C V(t)},
$$

where $V(t)=\int_{0}^{t}\left(\|\nabla v(\cdot, \tau)\|_{L^{\infty}}+\left\|\nabla u^{\alpha}(\cdot, \tau)\right\|_{L^{\infty}}\right) d \tau$. Hence by (3.2)

$$
\begin{aligned}
\left\|w^{\alpha}(\cdot, t)\right\|_{L^{2}} & \leq C \alpha^{3 / 2}\left\|q^{i n}\right\|_{\dot{B}_{2, \infty}^{1 / 2}}\left\|\nabla v^{i n}\right\|_{L^{\infty}} e^{C t} e^{C\left\|\nabla v^{i n}\right\|_{L^{\infty}} e^{C t}} \\
& \leq \alpha^{3 / 2} C e^{C e^{C t}}
\end{aligned}
$$

where $C$ depends only on the initial data $q^{i n}$.

Acknowledgements This work was supported in part by the BSF grant No. 2004271, the ISF grant No. 120/06, and the NSF grant No. DMS-0708832.

Open Access This article is distributed under the terms of the Creative Commons Attribution Noncommercial License which permits any noncommercial use, distribution, and reproduction in any medium, provided the original author(s) and source are credited.

\section{Appendix}

\section{A.1 Global Regularity of Contour Dynamics- $\alpha$ Equation}

We consider the vortex patch problem, i.e., a system in which the initial vorticity $q^{i n}$ is proportional to the characteristic function of a bounded domain $\Omega^{i n}, q^{i n}=q_{0} \chi_{\Omega^{i n}}$, under the evolution of the Euler- $\alpha$ equations (1.5). Due to the conservation of the two-dimensional Euler- $\alpha$ vorticity along particle trajectories for such an initial data (in fact, it is enough for the initial vorticity to be in the space of Radon measures in $\mathbb{R}^{2}$, see [65]), the vorticity $q^{\alpha}(t)$ remains a characteristic function of an evolving in time domain $\Omega^{\alpha}(t)$. In this section we present the result which states that the boundary of a vortex patch evolving under Euler- $\alpha$ equations (1.5) remains as smooth, for all time, as initially boundary, provided the latter is smooth enough in a sense specified in Theorem A.1 below.

In two dimensions the evolution of the boundary $x(\sigma, t)$ of a vortex patch under the Euler- $\alpha$ equation (1.5) is given by

$$
\begin{aligned}
\frac{\partial x}{\partial t}(\sigma, t) & =-q_{0} \int_{S^{1}} \Psi^{\alpha}\left(\left|x(\sigma, t)-x\left(\sigma^{\prime}, t\right)\right|\right) \frac{\partial x}{\partial \sigma}\left(\sigma^{\prime}, t\right) d \sigma^{\prime}, \\
x(\sigma, 0) & =x^{i n}(\sigma),
\end{aligned}
$$

where

$$
\Psi^{\alpha}(r)=\frac{1}{2 \pi}\left[K_{0}\left(\frac{r}{\alpha}\right)+\log r\right],
$$


and $K_{0}$ denote the modified Bessel functions of the second kind of order zero. As we mentioned before, for details on Bessel functions, see, e.g., [79]. The integro-differential equation (A.1) is an analogue of the so-called contour dynamics (CD) equation [58, 81], describing the evolution of the boundary of vortex patch evolving by means of the Euler equations (1.3):

$$
\begin{aligned}
\frac{\partial x}{\partial t}(\sigma, t) & =-\frac{q_{0}}{2 \pi} \int_{S^{1}} \log \left(\left|x(\sigma, t)-x\left(\sigma^{\prime}, t\right)\right|\right) \frac{\partial x}{\partial \sigma}\left(\sigma^{\prime}, t\right) d \sigma^{\prime}, \\
x(\sigma, 0) & =x^{i n}(\sigma) .
\end{aligned}
$$

The CD- $\alpha$ equation (A.1) is derived following arguments similar to those used in the Euler case, see [58, 81].

We show that CD- $\alpha$ equation (A.1) is well-posed in the space of Lipschitz functions and in the Hölder space $C^{n, \beta}, n \geq 1$, which is the space of $n$-times differentiable functions with Hölder continuous $n^{\text {th }}$ derivative. Let us first describe the Hölder space $C^{n, \beta}\left(S^{1} \subset \mathbb{R} ; \mathbb{R}^{2}\right)$, $\beta \in(0,1]$, which is the space of functions $x: S^{1} \subset \mathbb{R} \rightarrow \mathbb{R}^{2}$, with a finite norm

$$
\|x\|_{n, \beta}=\sum_{k=0}^{n}\left\|\frac{d^{k}}{d \sigma^{k}} x\right\|_{C^{0}\left(S^{1}\right)}+\left|\frac{d^{n}}{d \sigma^{n}} x\right|_{\beta},
$$

where

$$
\|x\|_{C^{0}\left(S^{1}\right)}=\sup _{\sigma \in S^{1}}|x(\sigma)|
$$

and $|\cdot|_{\beta}$ is the Hölder semi-norm

$$
|x|_{\beta}=\sup _{\substack{\sigma, \sigma^{\prime} \in S^{1} \\ \sigma \neq \sigma^{\prime}}} \frac{\left|x(\sigma)-x\left(\sigma^{\prime}\right)\right|}{\left|\sigma-\sigma^{\prime}\right|^{\beta}} .
$$

The Lipschitz space $\operatorname{Lip}\left(S^{1}\right)$ is the $C^{0,1}$ space, that is, with the finite norm $\|x\|_{\operatorname{Lip}\left(S^{1}\right)}=$ $\|x\|_{C^{0}\left(S^{1}\right)}+|x|_{1}$. We also use the notation

$$
|x|_{*}=\inf _{\substack{\sigma, \sigma^{\prime} \in S^{1} \\ \sigma \neq \sigma^{\prime}}} \frac{\left|x(\sigma)-x\left(\sigma^{\prime}\right)\right|}{\left|\sigma-\sigma^{\prime}\right|} .
$$

We consider the CD- $\alpha$ equation (A.1) as an evolution functional equation on either one of the following Banach spaces: Lip, $C^{1, \beta}, \beta \in[0,1], C^{2, \gamma}, \gamma \in[0,1)$, or $C^{n, \theta}, n \geq 3$, $\theta \in(0,1)$. We have the following result

Theorem A.1 Let $V$ be either one of the following spaces: $\operatorname{Lip}\left(S^{1}\right)$, or $C^{1, \beta}\left(S^{1}\right), \beta \in$ $[0,1]$, or $C^{2, \gamma}\left(S^{1}\right), \gamma \in[0,1)$, or $C^{n, \theta}\left(S^{1}\right), n \geq 3, \theta \in(0,1)$. Let $x^{\text {in }} \in V \cap\left\{|x|_{*}>0\right\}$, then there exists a unique solution $x \in C^{1}\left((-\infty, \infty) ; V \cap\left\{|x|_{*}>0\right\}\right)$ of (A.1) with initial value $x(\sigma, 0)=x^{\text {in }}(\sigma)$. In particular, if $x_{0} \in C^{\infty}\left(S^{1}\right) \cap\left\{|x|_{*}>0\right\}$ then $x \in$ $C^{1}\left((-\infty, \infty) ; C^{\infty}\left(S^{1}\right) \cap\left\{|x|_{*}>0\right\}\right)$.

We remark that, although the kernel $\Psi^{\alpha}$ and its first derivative $D \Psi^{\alpha}$ are continuous bounded functions, its higher derivatives $D^{m} \Psi^{\alpha}, m \geq 2$, are unbounded near the origin, 
and the chord arc condition $|x|_{*}>0$, which implies simple curves, allows us to show the integrability of the relevant terms.

We only sketch the main steps of the proof, since it is in the spirit of $[4,5]$ and $[58$, Chap. 8], which can be consulted for details of such a proof. In [5] we show the wellposedness of vortex sheet problem for Euler- $\alpha$ equations, and it contains various estimates involving the derivatives of the kernel $\Psi^{\alpha}$, and [58, Chap. 8] describes the proof of the original vortex patch problem in the Euler equations case.

The following are the main steps involved in the proof of Theorem A.1. In the first step, we apply the Contraction Mapping Principle to the CD- $\alpha$ equation (A.1) to prove the short time existence and uniqueness of solutions in the appropriate space of functions. Next, we derive an a priori bound for the controlling quantity for continuing the solution for all time. At step three one can extend the result for higher derivatives, using the estimates derived in [5] and [58, Chap. 8].

\section{A.1.1 Local Existence of Contour Dynamics- $\alpha$ Equation}

Next we show the local existence and uniqueness of solutions in the Lipschitz space, the details and the estimates in other appropriate spaces can be done in the same spirit following arguments presented in [5] and [58, Chap. 8]. First we recall some properties of the kernel $\Psi^{\alpha}$, see also (3.4)-(3.5). For $\frac{r}{\alpha} \rightarrow 0$

$$
\Psi^{\alpha}(r)=\frac{1}{2 \pi} \log \alpha+O(1)
$$

and for large $\frac{r}{\alpha}$

$$
\Psi^{\alpha}(r)=O(\log r) .
$$

Also, $D \Psi^{\alpha}$ is bounded for all $r \in[0, \infty)$,

$$
D \Psi^{\alpha}(r)=O\left(\frac{1}{\alpha}\right)
$$

where in the big $O$ the constants are independent of $\alpha$. To apply the Contraction Mapping Principle to the CD- $\alpha$ equation (A.1) we first prove the following result:

Proposition A.2 Let $1<M<\infty$, and let $K^{M}$ be the set

$$
K^{M}=\left\{x \in \operatorname{Lip}\left(S^{1}\right):\|x\|_{\text {Lip }}<M,|x|_{*}>\frac{1}{M}\right\} .
$$

Then the mapping

$$
x(\Gamma) \mapsto u(x(\Gamma))=\int_{S^{1}} K^{\alpha}\left(x(\Gamma)-x\left(\Gamma^{\prime}\right)\right) d \Gamma^{\prime}
$$

defines a locally Lipschitz continuous map from $K^{M}$, equipped with the topology induced by the $\|\cdot\|_{\text {Lip }}$ norm, into Lip.

Proof We start by showing that $u(x(\sigma))$ maps $K^{M}$ into Lip. Let $x \in K^{M}$. By (A.3) and (A.4) we have

$$
|u(x(\sigma))| \leq q_{0} \int_{S^{1}} \Psi^{\alpha}\left(\left|x(\sigma)-x\left(\sigma^{\prime}\right)\right|\right)\left|\frac{d x}{d \sigma}\left(\sigma^{\prime}\right)\right| d \sigma^{\prime}
$$




$$
\leq C q_{0}(\log \alpha+1+\log M)|x|_{1} .
$$

To show Lipschitz continuity of $u(x(\sigma))$ we use that by mean value theorem and (A.5), we have that for $x\left(\sigma^{\prime \prime}\right) \in B(x(\sigma),|x(\sigma)-x(\bar{\sigma})|)$, the ball centered at $x(\sigma)$ with the radius $|x(\sigma)-x(\bar{\sigma})|$,

$$
\begin{aligned}
\left|\Psi^{\alpha}\left(\left|x(\sigma)-x\left(\sigma^{\prime}\right)\right|\right)-\Psi^{\alpha}\left(\left|x(\bar{\sigma})-x\left(\sigma^{\prime}\right)\right|\right)\right| & \leq D \Psi^{\alpha}\left(\left|x\left(\sigma^{\prime \prime}\right)-x\left(\sigma^{\prime}\right)\right|\right)|x(\sigma)-x(\bar{\sigma})| \\
& \leq \frac{C}{\alpha}|x(\sigma)-x(\bar{\sigma})|,
\end{aligned}
$$

and hence

$$
\begin{aligned}
& |u(x(\sigma))-u(x(\bar{\sigma}))| \\
& \quad \leq q_{0} \int_{S^{1}}\left|\Psi^{\alpha}\left(\left|x(\sigma)-x\left(\sigma^{\prime}\right)\right|\right)-\Psi^{\alpha}\left(\left|x(\bar{\sigma})-x\left(\sigma^{\prime}\right)\right|\right)\right|\left|\frac{d x}{d \sigma}\left(\sigma^{\prime}\right)\right| d \sigma^{\prime} \\
& \quad \leq \frac{C}{\alpha} q_{0}|x|_{1}|x(\sigma)-x(\bar{\sigma})| .
\end{aligned}
$$

Now, we show that $u(x)$ is locally Lipschitz continuous on $K^{M}$. It is enough to prove that for $x \in K^{M}, y \in \operatorname{Lip}\left(S^{1}\right)$

$$
\left\|D_{x} u(x) y\right\|_{\text {Lip }} \leq C\left(\frac{1}{\alpha}, M,\|x\|_{\text {Lip }}\right)\|y\|_{\text {Lip }} .
$$

Let $x \in K^{M}, y \in H^{1}\left(S^{1}\right)$, we compute

$$
\begin{aligned}
& D_{x} u(x(\sigma)) y(\sigma) \\
&=\left.\frac{d}{d \varepsilon} u(x(\sigma)+\varepsilon y(\sigma))\right|_{\varepsilon=0} \\
&=-q_{0} \int_{S^{1}} D \Psi^{\alpha}\left(\left|x(\sigma)-x\left(\sigma^{\prime}\right)\right|\right) \frac{\left(x(\sigma)-x\left(\sigma^{\prime}\right)\right) \cdot\left(y(\sigma)-y\left(\sigma^{\prime}\right)\right)}{\left|x(\sigma)-x\left(\sigma^{\prime}\right)\right|} \frac{d x}{d \sigma}\left(\sigma^{\prime}\right) d \sigma^{\prime} \\
&-q_{0} \int_{S^{1}} \Psi^{\alpha}\left(\left|x(\sigma)-x\left(\sigma^{\prime}\right)\right|\right) \frac{d y}{d \sigma}\left(\sigma^{\prime}\right) d \sigma^{\prime} \\
&= F_{1}(x(\sigma)) y(\sigma)+F_{2}(x(\sigma)) y(\sigma) .
\end{aligned}
$$

Next we show (A.9). To estimate the $C^{0}$ norm we use (A.5) for $F_{1}$

$$
\begin{aligned}
\left|F_{1}(x(\sigma)) y(\sigma)\right| & \leq q_{0} \int_{S^{1}} D \Psi^{\alpha}\left(\left|x(\sigma)-x\left(\sigma^{\prime}\right)\right|\right)\left|y(\sigma)-y\left(\sigma^{\prime}\right)\right|\left|\frac{d x}{d \sigma}\left(\sigma^{\prime}\right)\right| d \sigma^{\prime} \\
& \leq \frac{C}{\alpha} q_{0}\|y\|_{C^{0}}|x|_{1}
\end{aligned}
$$

and (A.3) and (A.4) for $F_{2}$

$$
\begin{aligned}
\left|F_{2}(x(\sigma)) y(\sigma)\right| & \leq q_{0} \int_{S^{1}}\left|\Psi^{\alpha}\left(\left|x(\sigma)-x\left(\sigma^{\prime}\right)\right|\right)\right|\left|\frac{d y}{d \sigma}\left(\sigma^{\prime}\right)\right| d \sigma^{\prime} \\
& \leq C q_{0}(\log \alpha+1+\log M)|y|_{1} .
\end{aligned}
$$


Next we show Lipschitz continuity of $D_{x} u(x(\sigma)) y(\sigma)$. For $F_{2}$ one uses (A.8). For $F_{1}$ we have

$$
\begin{aligned}
& \left|F_{1}(x(\sigma)) y(\sigma)-F_{1}(x(\bar{\sigma})) y(\bar{\sigma})\right| \\
& \leq q_{0} \int_{S^{1}}\left|D \Psi^{\alpha}\left(\left|x(\sigma)-x\left(\sigma^{\prime}\right)\right|\right)-D \Psi^{\alpha}\left(\left|x(\bar{\sigma})-x\left(\sigma^{\prime}\right)\right|\right)\right|\left|y(\sigma)-y\left(\sigma^{\prime}\right)\right| \frac{d x}{d \sigma}\left(\sigma^{\prime}\right) \mid d \sigma^{\prime} \\
& \quad+2 q_{0} \int_{S^{1}} D \Psi^{\alpha}\left(\left|x(\bar{\sigma})-x\left(\sigma^{\prime}\right)\right|\right)|x(\bar{\sigma})-x(\sigma)| \frac{\left|y(\sigma)-y\left(\sigma^{\prime}\right)\right|}{\left|x(\bar{\sigma})-x\left(\sigma^{\prime}\right)\right|}\left|\frac{d x}{d \sigma}\left(\sigma^{\prime}\right)\right| d \sigma^{\prime} \\
& \quad+q_{0} \int_{S^{1}} D \Psi^{\alpha}\left(\left|x(\bar{\sigma})-x\left(\sigma^{\prime}\right)\right|\right)|y(\sigma)-y(\bar{\sigma})|\left|\frac{d x}{d \sigma}\left(\sigma^{\prime}\right)\right| d \sigma^{\prime} \\
& =I_{1}+I_{2}+I_{3} .
\end{aligned}
$$

For $I_{1}$, by the mean value theorem, (3.5) and due to the fact that $|x|_{*}>\frac{1}{M}$, we have that for $\sigma^{\prime \prime} \in S^{1}$ and such that $x\left(\sigma^{\prime \prime}\right) \in B(x(\sigma),|x(\sigma)-x(\bar{\sigma})|)$

$$
\begin{aligned}
\mid D & \Psi^{\alpha}\left(\left|x(\sigma)-x\left(\sigma^{\prime}\right)\right|\right)-D \Psi^{\alpha}\left(\left|x(\bar{\sigma})-x\left(\sigma^{\prime}\right)\right|\right) \mid \\
& \leq\left|D^{2} \Psi^{\alpha}\left(\left|x\left(\sigma^{\prime \prime}\right)-x\left(\sigma^{\prime}\right)\right|\right)\right||x(\sigma)-x(\bar{\sigma})| \\
& \leq\left(\frac{1}{4 \pi} \frac{1}{\alpha^{2}}\left|\log \frac{\left|x\left(\sigma^{\prime \prime}\right)-x\left(\sigma^{\prime}\right)\right|}{\alpha}\right|+\frac{C}{\alpha^{2}}\right)|x(\sigma)-x(\bar{\sigma})| \\
& \leq C(M) \frac{1}{\alpha^{2}}|\sigma-\bar{\sigma}|\left(\left|\log \left(\frac{\left|\sigma^{\prime \prime}-\sigma^{\prime}\right|}{\alpha}\right)\right|+1\right) .
\end{aligned}
$$

Therefore,

$$
\begin{aligned}
I_{1} & \leq|\sigma-\bar{\sigma}| C(M) \frac{1}{\alpha^{2}} q_{0}\|y\|_{C^{0}}|x|_{1} \int_{S^{1}}\left(\left|\log \left(\frac{\left|\sigma^{\prime \prime}-\sigma^{\prime}\right|}{\alpha}\right)\right|+1\right) d \sigma^{\prime} \\
& \leq C\left(M, \frac{1}{\alpha}, q_{0}\right)\|y\|_{C^{0}}|\sigma-\bar{\sigma}| .
\end{aligned}
$$

For $I_{2}$ and $I_{3}$ we use (A.5) and $|x|_{*}>\frac{1}{M}$ to obtain

$$
I_{2}, I_{3} \leq C\left(M, \frac{1}{\alpha}, q_{0}\right)|y|_{1}|\sigma-\bar{\sigma}| .
$$

Proposition A.2 implies the local existence and uniqueness of solutions:

Proposition A.3 Let $K^{M}=\left\{x \in \operatorname{Lip}\left(S^{1}\right):\|x\|_{\text {Lip }}<M,|x|_{*}>\frac{1}{M}\right\}$ and let $x_{0} \in \operatorname{Lip}\left(S^{1}\right) \cap$ $\left\{|x|_{*}>0\right\}$, then for any $M, 1<M<\infty$, such that $x_{0} \in K^{M}$, there exists a time $T(M)$, such that the system (A.1) has a unique local solution $x \in C^{1}\left((-T(M), T(M)) ; K^{M}\right)$.

\section{A.1.2 Global Existence of Contour Dynamics- $\alpha$ Equation}

To show the global existence of the CD- $\alpha$ equation, we assume by contradiction, that $T_{\max }<\infty$, where $\left[0, T_{\max }\right)$ is the maximal interval of existence, and hence the solution leaves in a finite time the open set $K^{M}$, for all $M>1$, that is, $\limsup _{t \rightarrow T_{\max }^{-}}\|x\|_{V}=\infty$ or 
$\lim \sup _{t \rightarrow T_{\max }^{-}} \frac{1}{|x(\cdot, t)|_{*}}=\infty$. Therefore, if we show global bounds on $\frac{1}{|x(\cdot, t)|_{*}}$ and $\|x(\cdot, t)\|_{V}$ in $\left[0, T_{\max }\right)$, we obtain a contradiction to the blow-up, and thus the obtained local solutions can be continued for all time. The result extends to negative times as well.

To control the quantities $\frac{1}{|x(\cdot, t)|_{*}}$ and $\|x(\cdot, t)\|_{V}$ one needs to bound $\int_{0}^{T_{\max }} \| \nabla_{x} u^{\alpha}(x(\cdot, t)$, $t) \|_{L^{\infty}} d t$. Next proposition shows the bound on $\left\|\nabla u^{\alpha}\right\|_{L^{\infty}}$ for the vortex patch initial data.

Proposition A.4 Let $q^{i n} \in L^{1}\left(\mathbb{R}^{2}\right) \cap L^{\infty}\left(\mathbb{R}^{2}\right)$ then

$$
\left\|\nabla_{x} u^{\alpha}(x(\cdot, t), t)\right\|_{L^{\infty}} \leq C\left(\frac{1}{\alpha}\right)\left(\left\|q^{i n}\right\|_{L^{\infty}}+\left\|q^{i n}\right\|_{L^{1}}\right) .
$$

Proof We write

$$
\begin{aligned}
\nabla_{x} u^{\alpha}(x, t) & =\int_{\mathbb{R}^{2}} \nabla K^{\alpha}(x-y) q^{\alpha}(y, t) d y \\
& =\int_{|x-y|<\alpha}+\int_{|x-y| \geq \alpha}=I_{1}+I_{2} .
\end{aligned}
$$

Using (3.5) we obtain

$$
\begin{aligned}
I_{1} & \leq C\left\|q^{\alpha}(\cdot, t)\right\|_{L^{\infty}} \int_{0}^{\alpha}\left|D^{2} \Psi^{\alpha}(r)\right| r d r \\
& \leq C\left\|q^{i n}\right\|_{L^{\infty}} \int_{0}^{\alpha} r\left|\frac{1}{4 \pi} \frac{1}{\alpha^{2}} \log \frac{r}{\alpha}+\frac{C}{\alpha^{2}}\right| d r \\
& \leq C\left\|q^{i n}\right\|_{L^{\infty}}
\end{aligned}
$$

and

$$
\begin{aligned}
I_{2} & \leq \sup _{|x-y| \geq \alpha}\left|\nabla K^{\alpha}(x-y)\right| \int_{\mathbb{R}^{2}} q^{\alpha}(y, t) d y \\
& \leq C \frac{1}{\alpha^{2}}\left\|q^{i n}\right\|_{L^{1}} .
\end{aligned}
$$

\section{References}

1. Abidi, H., Danchin, R.: Optimal bounds for the inviscid limit of Navier-Stokes equations. Asymptot. Anal. 38, 35-46 (2004)

2. Bardina, J., Ferziger, J.H., Reynolds, W.C.: Improved subgrid-scale models for large-eddy simulation. AIAA J. 80-1357 (1980)

3. Bardos, C.: Existence et unicité de la solution de l'équation d'Euler en dimension deux. J. Math. Anal. Appl. 40, 769-790 (1972)

4. Bardos, C., Linshiz, J.S., Titi, E.S.: Global regularity for a Birkhoff-Rott- $\alpha$ approximation of the dynamics of vortex sheets of the 2D Euler equations. Physica D 237, 1905-1911 (2008)

5. Bardos, C., Linshiz, J.S., Titi, E.S.: Global regularity and convergence of a Birkhoff-Rott- $\alpha$ approximation of the dynamics of vortex sheets of the 2D Euler equations. Commun. Pure Appl. Math. (2009, to appear)

6. Bardos, C., Titi, E.S.: Euler equations of incompressible ideal fluids. Usp. Mat. Nauk 62, 5-46 (2007)

7. Beale, J.T., Kato, T., Majda, A.: Remarks on the breakdown of smooth solutions for the 3-D Euler equations. Commun. Math. Phys. 94, 61-66 (1984)

8. Bergh, J., Löfström, J.: Interpolation Spaces. An Introduction. Grundlehren der Mathematischen Wissenschaften, vol. 223. Springer, Berlin (1976) 
9. Berselli, L.C., Iliescu, T., Layton, W.J.: Mathematics of Large Eddy Simulation of Turbulent Flows, Scientific Computation. Springer, Berlin (2006)

10. Bertozzi, A.L., Constantin, P.: Global regularity for vortex patches. Commun. Math. Phys. 152, 19-28 (1993)

11. Busuioc, A.V., Ratiu, T.S.: Some remarks on a certain class of axisymmetric fluids of differential type. Physica D 191, 106-120 (2004)

12. Cao, C., Holm, D.D., Titi, E.S.: On the Clark- $\alpha$ model of turbulence: global regularity and long-time dynamics. J. Turbul. 6, 20 (2005) (electronic)

13. Cao, Y., Lunasin, E., Titi, E.S.: Global well-posedness of the three-dimensional viscous and inviscid simplified Bardina turbulence models. Commun. Math. Sci. 4, 823-848 (2006)

14. Cao, Y., Titi, E.S.: On the rate of convergence of the two-dimensional $\alpha$-models of turbulence to the Navier-Stokes equations. Numer. Funct. Anal. Optim. (2009, to appear)

15. Chemin, J.-Y.: Persistance de structures géométriques dans les fluides incompressibles bidimensionnels. Ann. Sci. École Norm. Super. 26(4), 517-542 (1993)

16. Chen, S., Foias, C., Holm, D.D., Olson, E., Titi, E.S., Wynne, S.: Camassa-Holm equations as a closure model for turbulent channel and pipe flow. Phys. Rev. Lett. 81, 5338-5341 (1998)

17. Chen, S., Foias, C., Holm, D.D., Olson, E., Titi, E.S., Wynne, S.: The Camassa-Holm equations and turbulence. Physica D 133, 49-65 (1999)

18. Chen, S., Foias, C., Holm, D.D., Olson, E., Titi, E.S., Wynne, S.: A connection between the CamassaHolm equations and turbulent flows in channels and pipes. Phys. Fluids 11, 2343-2353 (1999). The International Conference on Turbulence (Los Alamos, NM, 1998)

19. Chen, L., Guenther, R.B., Kim, S., Thomann, E.A., Waymire, E.C.: A rate of convergence for the LANS $\alpha$ regularization of Navier-Stokes equations. J. Math. Anal. Appl. 348, 637-649 (2008)

20. Chen, S., Holm, D.D., Margolin, L.G., Zhang, R.: Direct numerical simulations of the Navier-Stokes alpha model. Physica D 133, 66-83 (1999)

21. Chepyzhov, V.V., Titi, E.S., Vishik, M.I.: On the convergence of solutions of the Leray- $\alpha$ model to the trajectory attractor of the 3D Navier-Stokes system. Discrete Contin. Dyn. Syst. 17, 481-500 (2007)

22. Cheskidov, A., Holm, D.D., Olson, E., Titi, E.S.: On a Leray- $\alpha$ model of turbulence. Proc. R. Soc. Lond., Ser. A, Math. Phys. Eng. Sci. 461, 629-649 (2005)

23. Clark, R.A., Ferziger, J.H., Reynolds, W.C.: Evaluation of subgrid-scale models using an accurately simulated turbulent flow. J. Fluid Mech. 91, 1-16 (1979)

24. Constantin, P.: Note on loss of regularity for solutions of the 3-D incompressible Euler and related equations. Commun. Math. Phys. 104(2), 311-326 (1986)

25. Constantin, P.: An Eulerian-Lagrangian approach to the Navier-Stokes equations. Commun. Math. Phys. 216, 663-686 (2001)

26. Constantin, P.: On the Euler equations of incompressible fluids. Bull. Am. Math. Soc. (N.S.) 44, 603-621 (2007) (electronic)

27. Constantin, P., Wu, J.: Inviscid limit for vortex patches. Nonlinearity 8, 735-742 (1995)

28. Dunn, J.E., Fosdick, R.L.: Thermodynamics, stability, and boundedness of fluids of complexity 2 and fluids of second grade. Arch. Ration. Mech. Anal. 56, 191-252 (1974)

29. Dunn, J.E., Rajagopal, K.R.: Fluids of differential type: critical review and thermodynamic analysis. Int. J. Eng. Sci. 33, 689-729 (1995)

30. Foias, C., Holm, D.D., Titi, E.S.: The Navier-Stokes-alpha model of fluid turbulence. Physica D 152/153, 505-519 (2001)

31. Foias, C., Holm, D.D., Titi, E.S.: The three dimensional viscous Camassa-Holm equations, and their relation to the Navier-Stokes equations and turbulence theory. J. Dyn. Differ. Equ. 14, 1-35 (2002)

32. Geurts, B.J., Holm, D.D.: Regularization modeling for large-eddy simulation. Phys. Fluids 15, L13-L16 (2003)

33. Geurts, B.J., Holm, D.D.: Leray and LANS- $\alpha$ modelling of turbulent mixing. J. Turbul. 7, 1-33 (2006)

34. Geurts, B., Kuczaj, A., Titi, E.S.: Regularization modeling for large-eddy simulation of homogeneous isotropic decaying turbulence. J. Phys. A, Math. Theor. 41, 344008 (2008) (29pp)

35. Grafakos, L.: Classical and Modern Fourier Analysis. Pearson Education, Upper Saddle River (2004)

36. Holm, D.D.: Variational principles for Lagrangian-averaged fluid dynamics. J. Phys. A 35, 679-688 (2002)

37. Holm, D.D., Marsden, J.E., Ratiu, T.S.: The Euler-Poincaré equations and semidirect products with applications to continuum theories. Adv. Math. 137, 1-81 (1998)

38. Holm, D.D., Marsden, J.E., Ratiu, T.S.: Euler-Poincaré models of ideal fluids with nonlinear dispersion. Phys. Rev. Lett. 80, 4173-4176 (1998)

39. Holm, D.D., Nadiga, B.T.: Modeling mesoscale turbulence in the barotropic double-gyre circulation. J. Phys. Oceanogr. 33, 2355-2365 (2003) 
40. Holm, D.D., Titi, E.S.: Computational models of turbulence: the LANS-alpha model and the role of global analysis, SIAM News 38 (2005)

41. Hou, T.Y., Li, C.: On global well-posedness of the Lagrangian averaged Euler equations. SIAM J. Math. Anal. 38, 782-794 (2006) (electronic)

42. Iftimie, D.: Remarques sur la limite $\alpha \rightarrow 0$ pour les fluides de grade 2. C. R. Math. Acad. Sci. Paris 334, 83-86 (2002)

43. Iftimie, D.: Remarques sur la limite $\alpha \rightarrow 0$ pour les fluides de grade 2. In: Nonlinear Partial Differential Equations and Their Applications. Collège de France Seminar, vol. XIV, Paris, 1997/1998. Stud. Math. Appl., vol. 31, pp. 457-468. North-Holland, Amsterdam (2002)

44. Ilyin, A.A., Lunasin, E., Titi, E.S.: A Modified-Leray- $\alpha$ subgrid scale model of turbulence. Nonlinearity 19, 879-897 (2006)

45. Jiu, Q., Niu, D., Titi, E.S., Xin, Z.: Axisymmetric Euler- $\alpha$ equations without swirl: existence, uniqueness, and Radon measure valued solutions. Preprint (2009)

46. Kato, T.: Nonstationary flows of viscous and ideal fluids in $\mathbf{R}^{3}$. J. Funct. Anal. 9, 296-305 (1972)

47. Kato, T.: Quasi-linear equations of evolution, with applications to partial differential equations. In: Spectral Theory and Differential Equations (Proc. Sympos., Dundee, 1974; dedicated to Konrad Jörgens). Lecture Notes in Math., vol. 448, pp. 25-70. Springer, Berlin (1975)

48. Kato, T.: Remarks on zero viscosity limit for nonstationary Navier-Stokes flows with boundary. In: Seminar on Nonlinear Partial Differential Equations, Berkeley, Calif., 1983. Math. Sci. Res. Inst. Publ., vol. 2, pp. 85-98. Springer, New York (1984)

49. Kato, T.: A remark on a theorem of C. Bardos on the 2D Euler equation. U. C. Berkeley, Preprint (1992)

50. Layton, W., Lewandowski, R.: A simple and stable scale-similarity model for large eddy simulation: energy balance and existence of weak solutions. Appl. Math. Lett. 16, 1205-1209 (2003)

51. Layton, W., Lewandowski, R.: On a well-posed turbulence model. Discrete Contin. Dyn. Syst. Ser. B 6, 111-128 (2006)

52. Lemarié-Rieusset, P.G.: Recent Developments in the Navier-Stokes Problem. Research Notes in Mathematics, vol. 431. Chapman \& Hall/CRC, Boca Raton (2002)

53. Lewandowski, R.: Vorticities in a LES model for 3D periodic turbulent flows. J. Math. Fluid. Mech. 8, 398-422 (2006)

54. Lichtenstein, L.: Über einige Existenzprobleme der Hydrodynamik homogener, unzusammendrückbarer, reibungsloser Flüssigkeiten und die Helmholtzschen Wirbelsätze. Math. Z. 23, 89-154 (1925)

55. Lichtenstein, L.: Über einige Existenzprobleme der Hydrodynamik. Math. Z. 32, 608-640 (1930)

56. Linshiz, J.S., Titi, E.S.: Analytical study of certain magnetohydrodynamic-alpha models. J. Math. Phys. 48, 065504 (2007) 28

57. Liu, X.F., Jia, H.Y.: Local existence and blowup criterion of the Langrangian averaged Euler equations in Besov spaces. Commun. Pure Appl. Anal. 7, 845-852 (2008)

58. Majda, A.J., Bertozzi, A.L.: Vorticity and Incompressible Flow. Cambridge Texts in Applied Mathematics, vol. 27. Cambridge University Press, Cambridge (2002)

59. Marchioro, C., Pulvirenti, M.: Mathematical Theory of Incompressible Nonviscous Fluids. Applied Mathematical Sciences, vol. 96. Springer, New York (1994)

60. Marsden, J.E., Shkoller, S.: The anisotropic Lagrangian averaged Euler and Navier-Stokes equations. Arch. Ration. Mech. Anal. 166, 27-46 (2003)

61. Masmoudi, N.: Remarks about the inviscid limit of the Navier-Stokes system. Commun. Math. Phys. 270, 777-788 (2007)

62. McGrath, F.J.: Nonstationary plane flow of viscous and ideal fluids. Arch. Ration. Mech. Anal. 27, 329348 (1967)

63. Mohseni, K., Kosović, B., Shkoller, S., Marsden, J.E.: Numerical simulations of the Lagrangian averaged Navier-Stokes equations for homogeneous isotropic turbulence. Phys. Fluids 15, 524-544 (2003)

64. Oliver, M.: The Langrangian averaged Euler equations as the short-time Inviscid limit of the NaiverStokes equations with Besov class data in $\mathbb{R}^{2}$. Commun. Pure Appl. Anal. 1(2), 221-235 (2002)

65. Oliver, M., Shkoller, S.: The vortex blob method as a second-grade non-Newtonian fluid. Commun. Part. Differ. Equ. 26, 295-314 (2001)

66. Peetre, J.: New Thoughts on Besov Spaces, Mathematics Department, Duke University, Durham, N.C., 1976. Duke University Mathematics Series, No. 1

67. Pietarila Graham, J., Holm, D., Mininni, P., Pouquet, A.: Three regularization models of the NavierStokes equations. Phys. Fluids 20, 035107 (2008)

68. Polyanin, A.D.: Handbook of Linear Partial Differential Equations for Engineers and Scientists. Chapman \& Hall/CRC, Boca Raton (2002)

69. Swann, H.S.G.: The convergence with vanishing viscosity of nonstationary Navier-Stokes flow to ideal flow in $R_{3}$. Trans. Am. Math. Soc. 157, 373-397 (1971) 
70. Taniuchi, Y.: Uniformly local $L^{p}$ estimate for 2-D vorticity equation and its application to Euler equations with initial vorticity in bmo. Commun. Math. Phys. 248, 169-186 (2004)

71. Temam, R., Wang, X.: The convergence of the solutions of the Navier-Stokes equations to that of the Euler equations. Appl. Math. Lett. 10, 29-33 (1997)

72. Torchinsky, A.: Real-Variable Methods in Harmonic Analysis. Pure and Applied Mathematics, vol. 123. Academic Press, Orlando (1986)

73. Triebel, H.: Theory of Function Spaces. Monographs in Mathematics, vol. 78. Birkhäuser, Basel (1983)

74. Truesdell, C.: Fluids of second grade regarded as fluids of convected elasticity. Phys. Fluids 8, 1936$1938(1965)$

75. Vishik, M.: Incompressible flows of an ideal fluid with vorticity in borderline spaces of Besov type. Ann. Sci. École Norm. Super. 32(4), 769-812 (1999)

76. Vishik, M.I., Titi, E.S., Chepyzhov, V.V.: Trajectory attractor approximations of the 3D Navier-Stokes system by a Leray- $\alpha$ model. Rus. Math. Dokl. 71, 92-95 (2005) (Translated from Russian)

77. Vishik, M.I., Titi, E.S., Chepyzhov, V.V.: On convergence of trajectory attractors of the 3D NavierStokes- $\alpha$ model as $\alpha$ approaches 0 . Sb. Math. 198, 1703-1736 (2007)

78. Wang, X.: A Kato type theorem on zero viscosity limit of Navier-Stokes flows. Indiana Univ. Math. J. 50, 223-241 (2001). Dedicated to Professors Ciprian Foias and Roger Temam (Bloomington, IN, 2000)

79. Watson, G.N.: A Treatise on the Theory of Bessel Functions. Cambridge Mathematical Library. Cambridge University Press, Cambridge (1995)

80. Yudovich, V.I.: Non-stationary flow of an ideal incompressible liquid. Zh. Vychisl. Mat. Mat. Fiz. 3, $1032-1066$ (1963)

81. Zabusky, N.J., Hughes, M.H., Roberts, K.V.: Contour dynamics for the Euler equations in two dimensions. J. Comput. Phys. 30, 96-106 (1979) 